\title{
Unraveling the Connections between Calreticulin and Myeloproliferative Neoplasms via Calcium Signalling
}

\author{
Amit Jaiswal ${ }^{1,2^{*}}$, Zhiguo Wang ${ }^{2}$, Xudong Zhu², Zhenyu Ju', \\ ${ }^{1}$ Faculty of Biological Sciences, Friedrich Schiller University, 07737 Jena, Germany \\ ${ }^{2}$ Institute of Ageing Research, School of Medicine, Hangzhou Normal University, Hangzhou \\ 311121, Zhejiang, China \\ ${ }^{3}$ Key Laboratory of Regenerative Medicine of Ministry of Education, Institute of Aging and \\ Regenerative Medicine, Jinan University, Guangzhou 510632, Guangdong, China
}

"Corresponding author:

AmitJaiswal,e-mail: amitjaiswal@yandex.com; amit.jaiswal@uni-jena.de ORCID ID: https://orcid.org/0000-0002-4778-5955 


\section{$\underline{\text { Abstract }}$}

Mutations in the form of insertions and deletions (INDEL) in the calreticulin gene lead to essential thrombocythemia which is characterized by the formation of thrombosis. However, the connection between calreticulin INDEL and essential thrombocythemia remains largely elusive. Through combined molecular dynamics simulation and in-vitro studies on the wild type and mutated isoforms of calreticulin, the mechanism underlying the calreticulin INDEL induced essential thrombocythemia was investigated at the molecular level. Our results demonstrate that mutations in exon-9 could lead to significant conformational variations of calreticulin structure and thereby reducing its interaction with calcium ions due to decreased electrostatic contributions. The consequence of mutations on calreticulin's structural integrity was revealed by identifying the key residues and their roles in calcium binding. Furthermore, mutations implemented by CRISPR-Cas9 in exon-9 showed diminished calcium signaling in HEK-293T cells, which agree well with our insilico findings. The current study might help in understanding of the interactions between calreticulin exon-9 INDEL and calcium ions mediated by the structural variations of calreticulin. The results provide useful information for designing novel therapeutic approaches targeting essential thrombocythemia.

\section{Keywords}

Calreticulin, Molecular Dynamics Simulation, CRIPSR-Cas9, Calcium Ion, GROMACS, Essential Thrombocythemia 


\section{Introduction}

Essential thrombocythemia (ET) is a pathological condition which is characterized by the formation of excessive mature blood cells leading to thrombosis [1]. ET belongs to a class of diseases known as myeloproliferative neoplasms (MPNs) which develop through neoplastic transformation of myeloid cells [2] . ET, polycythemia vera (PV), and primary myelofibrosis (PMF) are $B C R-A B L 1$ negative MPNs, whereas chronic myeloid leukemia (CML) is characterized by the presence of the $\mathrm{t}(9 ; 22)$, also known as the Philadelphia chromosome translocation, which results in the generation of the $B C R-A B L 1$ oncogene $[3,4]$. Studies have shown that almost all MPNs are associated with either a JAK2 V617F mutation or changes in JAK2 signaling, either directly or indirectly [5]. JAK2 drives CML by phosphorylating BCR-ABL1 and triggers 50-60\% of ET with myeloproliferative leukaemia virus oncogene $(M P L)(3-5 \%)$ and calreticulin $(C A L R)(30-40 \%)$ responsible for the rest of ETs [6,7]. Though CALR mutation has been identified as a marker of MPNs, the underlying molecular process remains largely unrevealed, intriguing us to further explore the mechanism of $C A L R$-mediated ETs at the molecular level.

Highly conserved CALR is a calcium-dependent chaperone that is localized in the lumen of endoplasmic reticulum (ER) [8] facilitating calcium $\left(\mathrm{Ca}^{2+}\right)$ homeostasis of the ER by balancing $\mathrm{Ca}^{2+}$ communication between ER, plasma membrane, mitochondria and nucleus [9]. Moreover, it has been shown that systemic $C A L R$ knock-out $(\mathrm{KO})$ is embryonic lethal due to impaired cardiogenesis while a conditional $\mathrm{KO}$ perturbs secondary messengers such as inositol 1,4,5-trisphosphate [10]. Structurally, CALR contains three domains: a global domain called N-domain responsible for chaperone-like functions, a proline-rich P-domain containing a flexible arm-like structure, and a Cterminal acidic domain comprising of a KDEL motif responsible for an ER retention signal [11, 12]. The KDEL sequence acts as a signature in other ER-resident chaperons to recognize and retrieve KDEL-containing proteins from ER-Golgi transport and vice versa [13]. 
Calcium homeostasis is maintained in the cell involving various calcium transport molecules, buffers and sensors that control free and bound calcium levels in different cellular compartments such as cytoplasm, nucleus, mitochondria and ER [14]. Apart from protein folding and transportation, the ER primarily functions as a calcium storage pool as many calcium related proteins reside on the ER membrane [15]. Accumulation of misfolded proteins in the ER can alter the calcium balance resulting in the development of many diseases $[16,17]$. Changes in the calcium concentration are regulated by extracellular calcium uptake or intracellular calcium release in order to initiate a calcium electrochemical gradient flow resulting in calcium signaling [18]. There are two groups of calcium-binding proteins, namely cytosolic and organellar which show a different pattern of affinity towards calcium binding. The cytosolic group binds calcium with high affinity and low capacity while the organellar group binds it with high capacity and low affinity [19]. All these processes are highly orchestrated in a very intricate manner and if disturbed, serious consequences may occur such as cardiac arrhythmia [20].

Although biochemical studies have provided plenty of information about $\operatorname{CALR}[5,21,22]$, very little is known about the C-domain, especially the molecular mechanism of the C-terminus related to calcium ions due to the lack of structural information. Here we aim to elucidate the molecular mechanism of $C A L R$ in the context of calcium homeostasis and ET by adopting structural bioinformatics and CRISPR-Cas9 approaches. Our molecular dynamics (MD) study suggests that loss of CALR C-terminal residues would disrupt the negative potential of exon-9 leading to inefficient calcium binding. We propose that calcium-overload is supposed to trigger an aberrant calcium signaling pathway, leading to persistent activation of platelets culminating in the clinical manifestation of thrombosis. Exon-9-mutant HEK-293T cells exhibited diminished cellular calcium fluorescence compared to WT cells, strongly supporting our computational findings. Overall, the current work provides mechanistic insights in the binding of $C A L R$ and calcium ions and also novel information for developing new therapeutics targeting ET. 


\section{$\underline{\text { Results }}$}

\section{Sequence and Structural Characterization of Calreticulin}

The gene locus of CALR is situated on chromosome 19 and is 417 amino acids long consisting of nine exons (Figure 1A and B). Functional domain analysis by Pfam revealed the presence of a single family present in two regions of calreticulin protein: one starting from 1 to 253 and the other one from 293 to 367 while Interproscan disclosed two protein families, namely the Glucanase domain family comprising residues 19 to 217 and a calreticulin protein family beginning from amino acid 201 to 315 . The Uniprot web-server showed the presence of three domains: a N-domain comprising amino acids 18-197, a P-domain from 198-308 and a C-terminal acidic domain from 309 to 417 as shown in Table 1 and Figure 1C.

Table 1. List of protein domains identified by Pfam, InterProScan and Uniprot web server

\begin{tabular}{c|ccc}
\hline Webserver used & \multicolumn{3}{|c}{ Domains identified } \\
\hline Pfam & $\begin{array}{c}\text { Calreticulin Protein } \\
\text { family }(1-253)\end{array}$ & $\begin{array}{c}\text { Calreticulin Protein } \\
\text { family (293-367) }\end{array}$ & NA \\
InterProScan & $\begin{array}{c}\text { Glucanase Domain } \\
\text { Family (19-217) }\end{array}$ & $\begin{array}{c}\text { Calreticulin Protein } \\
\text { family (201 - 315) }\end{array}$ & NA \\
& $\begin{array}{c}\text { N-Domian 18-197 } \\
\text { (Globular } \\
\text { Architecture) }\end{array}$ & $\begin{array}{c}\text { P-Domain 198-308 } \\
\text { (Proline rich domain) }\end{array}$ & $\begin{array}{c}\text { C-Domain 309-417 } \\
\text { (Acidic Architecture) }\end{array}$ \\
\hline
\end{tabular}

Multiple Sequence alignments of $C A L R$ to its homologous sequences in various other mammalian species using the Clustl-X algorithm showed the existence of highly conserved amino acids throughout the sequence with an exception of the C-terminal region in exon-9 leading to a charge variation. The last seven amino acids comprising of the KDEL motif in exon- 9 were also highly conserved portraying the importance of this region (Figure. S1). Lack of structure for full-length sequence in the protein data bank required us to use the I-TASSER web-server to generate full- 
length structures, reasoning that full-length models would provide a more complete understanding of all domain movements and dynamics arising due to mutations. In order to validate the structures, we performed a Ramachandran plot assessment which suggested that $80.3 \%$ of residues were present in the most favored region for the WT while $81 \%$ and $82 \%$ for Ins and Del variants/mutations, respectively (Figure S2). The structural quality score provided by Verify-3D proposed $85.37 \%, 80 \%, 83.75 \%$, and ERRAT proposed $69.14 \%, 82.59 \%, 76.70 \%$ for WT, Ins and Del, suggesting the reliability of the I-TASSER models for further studies (Table2).

Table 2. Structural Validation of generated 3D structures through Ramachandran plot assessment, Verify 3D and ERRAT scores

\begin{tabular}{|c|ccc|}
\hline & \multicolumn{3}{|c|}{ 3D Structural Assessment } \\
\hline $\begin{array}{c}\text { Residues in favored region } \\
(\%)\end{array}$ & WT & Ins & Del \\
\cline { 2 - 4 } $\begin{array}{c}\text { Residues in additionally } \\
\text { allowed region (\%) } \\
\text { Residues in generously } \\
\text { allowed region (\%) } \\
\text { Residues in disallowed } \\
\text { region (\%) }\end{array}$ & 17.7 & 81 & 82 \\
$\begin{array}{c}\text { Verify_3D score (\%) } \\
\text { ERRAT score (\%) }\end{array}$ & 1.7 & 16.5 & 16.6 \\
\hline
\end{tabular}

The selected models were then subjected to molecular dynamics simulation (MDS) using the GROMACS 4.5.6 package. All proteins: WT, Ins and Del were simulated in the presence and absence of calcium ions in order to decipher the structural changes governed by the effects of calcium. The parameters for the simulation system are stated in Table S2. A preliminary study of 
secondary structure using the PDBsum web-server gave a discernment about secondary structures. The number of strands, helices, helix-helix interactions and turns was higher in Del and Ins while $\beta$-turns were higher in the WT (Table S3). However, when WT-Ca was compared to Ins-Ca and Del-Ca, a higher number of $\beta$-strands and $\beta$-turns was found while a lower number of helices was observed in WT-Ca as compared to other forms (Table S3). The minimum and maximum potential energy (PE) was observed to be $-2732658 \mathrm{~kJ} / \mathrm{mol}$ and $-2720337 \mathrm{~kJ} / \mathrm{mol}$ for $\mathrm{WT},-3103612 \mathrm{~kJ} / \mathrm{mol}$ and $-3087532 \mathrm{~kJ} / \mathrm{mol}$ for Ins and $-3664817 \mathrm{~kJ} / \mathrm{mol}$ and $-3650847 \mathrm{~kJ} / \mathrm{mol}$ for Del counterparts while the kinetic energy (KE) analysis showed contrasting results: The WT had the lowest KE compared to mutated equivalents (Table S4). Pressure and enthalpy were higher in the WT compared to mutated counterparts as can be seen from Table S4.

\section{Mutations in Exon-9 display huge structural Aberrations in the C-Domain}

Three-dimensional structures of WT, Ins and Del were significantly dissimilar despite of the fact that all the proteins under investigation are identical from exon- 1 to exon- 8 . Mutated isoforms such as Ins and Del had a similar surface structure where the C-terminal region collapsed to form a continuous loop-like structure. The two helices in exon-9 overlap with each other while it is stretched out in an elongated conformation in the WT (Figure 2 A-C). To measure the conformational stability of the proteins, Root Mean Square Deviation (RMSD) profiles for protein backbone residues were generated. RMSD results presented in figure 2D show the trajectory of the WT to be stable after $42 \mathrm{~ns}$ whereas for Ins it took around 40ns while Del was unable to attain proper stability even after 50ns. The radius of gyration $(\mathrm{Rg})$ was calculated by comparing the protein backbone residues with the native structure in order to observe the compactness of the protein structures. The average Rg for the WT was $3.45 \mathrm{~nm}$ while that of Ins and Del was $2.79 \mathrm{~nm}$ and $2.54 \mathrm{~nm}$, respectively (Figure 2E). Residue fluctuation for each protein was also calculated throughout a 50ns timescale by computing Root Mean Square fluctuation (RMSF) as can be seen 
in figure 3A. RMSF results demonstrated the WT to have a huge fluctuating P-domain and a Cterminal domain ranging from 230-280 (0.5-2.8 nm) to 370-417 (1-2.3 nm) while in Ins the residue range was reduced between residues 210-290 (0.4-1.2 nm) and 370-420 (0.6-1.1 nm). In Del residues 220-290 showed an overall fluctuation which ranged from 0.6-1.2 nm. Together, given the results from RMSD, Rg and RMSF; the WT proved to be highly unstable, most likely because of the presence of an unstable $\mathrm{P}$-domain and C-domain.

Structures of WT and mutated genes (Ins and Del) after 50ns of simulation were structurally compared using structural superimposition which showed huge structural differences (Figure 5). The C-terminal region comprising of the KDEL motif of WT had an open form compared to mutated equivalents. The hinge region comprising of R366 - E369 in the C-domain of the WT had an open $\alpha$-helix conformation while in the mutated forms it was closed. The presence of a $\beta$-sheet was also visible in the extended arms of the P-domain ranging in the region K224-D226 and Q279-D281, respectively. Three-dimensional structural analysis showed that the $\mathrm{N}$-domain or the globular domain fluctuated less compared to the P-and C-terminal acidic domains in all the proteins (Figure 5A,B). A Root Mean Square Deviation (RMSD) score of $21.776 \AA$ A was observed between WT and Ins while $25.505 \AA$ between WT and Del (Figure4).

We then aimed to analyse the secondary structure formation through the simulation of 50ns and thus we performed DSSP (Define Secondary Structure of Proteins) by using the inbuilt DSSP tool in the GROMACS package. The DSSP results for WT, Ins and Del showed a significant difference. Residues 413 to 416 attained 3-helix configurations throughout the simulation while Ins and Del lost this configuration. Moreover, in WT residues 397 to 409 took a configuration of a $\beta$-turn after 40ns of a MD run and remained stable throughout the simulation, while in Ins and Del the aforementioned residues took a configuration of $\alpha$-helices and coils, respectively (FigureS3). The majority of residues ranging between 350-395 in the WT were an $\alpha$-helix and the same holds true for Ins and Del. However, Del formed an additional 5-helix structure from 380-384 indicating a 
structural difference from WT. The most crucial point of observation was that exon-9 residues which ranged between 396-410 and 413-416 showed the formation of a $\beta$-turn and a 3-helix throughout the simulation which was not present in both mutated proteins. We also observed differences in the residues ranging from 311-335 having $\beta$-sheet conformation in the WT but this had been greatly reduced in mutated proteins (Figure S3).

\section{The Presence of $\mathrm{Ca}^{++}$ions provides structural stability}

In the presence of calcium, the WT-Ca showed lower PE and higher KE compared to Ins-Ca and Del-Ca. (Table S4) while structural analysis revealed that the P- and C-domains held their unique structure quite firmly surrounded by calcium ions in the WT. In case of Ins-Ca and Del-Ca, the P- and C-domains merged together losing their structural symmetry. In addition, the number of calcium ions that interacted with the $\mathrm{C}$-terminus was much less in mutated proteins compared to the WT (Figure 4 A-C). RMSD analysis of protein backbone revealed that WT-Ca took only about 20ns to become stable and remained stable all the way through. In contrast, Ins-Ca and Del-Ca attained stability at around 45ns evidencing that WT in the presence of calcium had more firmness and stability compared to its mutated equivalents (Figure 4D).

Furthermore, WT-Ca had less structural integrity in comparison to Ins-Ca and Del-Ca, possibly because of non-functional disintegrated $\mathrm{N}$ - and C-terminal domains which remain intact in the mutants. The average Rg for WT-Ca was $2.99 \mathrm{~nm}$ while that of Ins-Ca and Del-Ca was $2.65 \mathrm{~nm}$ and $2.3 \mathrm{~nm}$ (Figure4E). RMSF results for WT-Ca ranged from residues 220-280 (0.5-2.1nm) to 370-417 (0.3-1.6 nm) while for Ins-Ca and Del-Ca the fluctuating residues resided between 220-290 (0.5-0.9 $\mathrm{nm})$ and 210-300 (0.3-0.5 nm), respectively (Figure 3B). The same was found in the region of 370417 except for the fact that in WT-Ca the fluctuations declined severely. Taken together with the results from RMSD, Rg and RMSF, we concluded that the majority of calcium ions anchors to the C-terminal region and imparts stability. 
Moreover, 3D structural imposition showed a deviation of $25.172 \AA$ between WT-Ca and Ins-Ca, while it was $20.776 \AA$ between WT-Ca and Del-Ca. These huge deviations suggest that calcium ions play a significant role in stabilizing the P- and C-domains significantly in WT (Figure.5 C-D). For the DSSP results, the WT showed similar signatures to its non-calcium counterparts except for an additional formation of a 3-helix in the region of 399-402, while in Ins-Ca and Del-Ca the majority of configurations was similar to the non-calcium counterparts. In addition, the residues ranging from amino acids 154-167 in Ins-Ca showed a coil configuration while WT-Ca revealed a $\beta$-sheet. Moreover, residues 153-155 showed a $\beta$-turn while residues 160 -162 only formed $\beta$-sheets and the remaining residues all formed exclusively coils (Figure S4).

\section{$\mathrm{Ca}^{++}$ions but not $\mathrm{Na}^{+}$show more binding Affinity_towards the C-terminal Region}

To determine structural differences, we compared the WT structures both in the presence and absence of calcium. The WT in a calcium environment attained stability just after 20ns while the WT in the presence of $\mathrm{Na}^{+}$took 45ns. Furthermore, the average Rg of WT-Ca was about $2.9 \mathrm{~nm}$ which remained stable while that of WT ranged between 3-4 $\mathrm{nm}$ and had yet to attain stability (Figures 2, 3 and 4). Finally, the RSMF results suggested the P-domain to be less fluctuating in WTCa than WT. Moreover, the C-terminal region comprising of the KDEL motif also showed a huge decline in fluctuation indicating the region to be more stable in the presence of calcium (Figure. 3). Furthermore, structural superposition of protein structures showed huge variations between WT and WT-Ca with a deviation of 23.41 $\AA$ especially in the P- and C-domains (Figure 6). Comparing the DSSP results for calcium equivalents, the WT showed similar signatures to WT-Ca with an exception by forming a 3-helix in the region of 399-402 (Figure S3). The secondary structures of Ins-Ca and Del-Ca were similar to the non-calcium counterparts suggesting that the presence of calcium could not disturb the structural stability in mutated proteins (Figure. S3 and S4). Residues 
208-209 and 293-294 in WT-Ca underwent conformational changes forming a $\beta$-bridge to a $\beta$-sheet which was absent in mutated proteins.

The total number of amino acids present in exon-9 in the WT is 66 while in Del and Ins it is 49 and 69 , respectively. The Protparam tool indicated that $75 \%, 38 \%$ and $18 \%$ of amino acids were negatively charged in the WT, Ins and Del counterparts suggesting that mutation (INDEL's) lead to the loss of negative amino acids thereby decreasing the entire negative potential (Figure S5A). The C-terminal region was highly unstable as evidenced by the Instability Index and IUPred 2 webserver [44] (Figures S5B and S5C). The percentage of distinct amino acids in exon-9 also varied a lot. The amount of amino acids like aspartic acid, glutamine, leucine, glycine and valine was significantly higher in the WT compared to mutants while levels of arginine, methionine and cysteine and proline were lower in the WT than in its mutated counterparts (Figure S5D) and the isoelectric point of exon-9 was much lower in the WT compared to the other two forms (Figure S5E). However, analysing the aliphatic index, we found that the WT protein had a much higher presence of aliphatic side chains (alanine, valine, isoleucine, and leucine), signifying an increase in the thermo-stability of exon-9 [45] . Atomic composition of exon-9 in the WT showed the presence of sulphur to be around $5 \%$ while in the mutational counterparts it was $45 \%$ each proposing the mutated proteins to be more susceptible to forming thiol $(\mathrm{S}-\mathrm{H})$ and disulfide bonds (S-S) (Figure S5F). It has been reported that methionine and cysteine are the only two natural amino acids which have sulphur atoms [46]. Thus, it appeared that the mutated proteins had a higher number of methionine and cysteine amino acids in exon-9 compared to the WT.

We then explored the calcium dynamics of calreticulin in the presence of exon-9. The Mindist tool from the Gromacs package informed us about the distance of calcium ions from exon- 9 throughout the simulation. The average minimum distance of calcium ions from exon-9 was found to be $3.7 \AA$ in WT-Ca and remained stable, while for the WT it was very chaotic as the variations ranged from $2-4 \AA$ and were unstable throughout the simulation (Figure7A). We also performed a H-bond 
analysis using the Gromacs tool to determine the difference between intra-molecular H-bonds within exon-9. To our surprise, a higher number of $\mathrm{H}$-bonds was observed in the presence of calcium ions suggesting that calcium ions provide structural stability to the exon-9 region of $C A L R$ (Figure 7B). Moreover, it is worth to note that $\mathrm{Na}^{+}$ions were unable to provide enough stability to balance the negative amino acids present in exon-9 indicating the importance of calcium ions. In order to study the electrostatic differences between the WT and mutated proteins, Adaptive PoissonBoltzmann Solver (ABPS) studies were conducted using Pymol software. The presence of calcium supported the folding of the C-terminal acidic domain and this fold remained stable throughout the MD run through increased electrostatic interaction. The electrostatic charges in mutated proteins were less due to mutations in exon- 9 while the presence of calcium influenced both $\mathrm{N}$-and $\mathrm{C}$-termini by increasing their electrostatic potential (Figures $7 \mathrm{C}$, D and S6).

Also, the mere presence of $\mathrm{Na}$ ions was not enough to effectively bind to the negative charges present in the $\mathrm{C}$-domain compared to $\mathrm{Ca}$ ions while for mutated genes the electrostatic potential was too low for calcium ions to bind. Overall, the WT protein in the presence of calcium ions showed higher electrostatic interactions with calcium ions that proteins derived from mutated genes (Figure 6 and S6).

\section{Interaction of Exon-9 with Calcium Ions stabilizes the N-Domain and P-Domain}

We aimed to examine whether the stabilization of the C-terminal domain could impart stability to the overall protein structure. To this end, we performed visual inspection of the $50^{\text {th }} \mathrm{ns}$ structures from the simulated trajectory. The extended arm-like structure in the P-domain remains stable due to the close association with the N-domain. Residues Gly140, His241 and Ile242 from the N- and P-domains interacted with each other by forming hydrogen bonds after 30ns of simulation, thereby providing anchorage to the P-domain (Figure 8A,B). This interaction was possible because of the presence of the $\beta$-bend and $\beta$-bridge in WT-Ca while this was lacking in mutated counterparts. 
Additionally, the N-domain and C-terminal domains also interacted with each other in the presence of calcium ions. Asp59 and Glu60 of the N-domain interacted with Glu386 and Glu389 of the Cterminal domain suggesting that calcium ions not only stabilize the $\mathrm{C}$-terminal region but also brings conformational changes to the $\mathrm{C}$-terminal region in order to interact with the $\mathrm{N}$-domain, thus providing overall stability. The $2 \mathrm{D}$ interaction plot between the above residues were cross-validated using Ligplot software [47] (Figure S7 A, B). The residue mobility scores for WT and WT-Ca indicated that the mobility of the residues of the P- and C-terminal domains in the WT was very high with a score of 300 while for WT-Ca the score was merely 5 suggesting a higher steadiness in WT-Ca (Figure 8C and D). Secondary structure comparison between WT and WT-Ca suggested that residues 138-140 and 241-242 hold a $\beta$-bend configuration in WT-Ca but not in WT. Moreover, the key residues Gly140-Ile242 undergo changes with time forming a $\beta$-bridge which makes the interaction more prominent. Furthermore, domain movement analysis by VMD in the form of porcupine plot described that the movement of the C-terminal domain in WT was inwards but outwards in WT-Ca as indicated by the arrows (Figure S8 A, B).

Next, we examined the exon-9 structural changes in WT and WT-Ca throughout the simulation period. Interactions of all the protein residues to calcium ions are shown in figure S9 and a full list of residues is shown in Table 3.

Table 3: List of WT residues interacting with calcium ions

\begin{tabular}{ccc}
\hline $\begin{array}{c}\text { Residue } \\
\text { name }\end{array}$ & $\begin{array}{c}\text { Residue } \\
\text { number }\end{array}$ & $\begin{array}{c}\text { Distance of residues from calcium ions } \\
(\mathcal{A})\end{array}$ \\
\hline Gly & 58 & 2.55 \\
Asp & 59 & 2.55 \\
Glu & 60 & 2.58 \\
Glu & 61 & 2.71 \\
Asp & 118 & 2.68 \\
\hline
\end{tabular}




\begin{tabular}{|c|c|c|}
\hline Asp & 121 & 2.53 \\
\hline Asp & 166 & 2.54 \\
\hline Glu & 167 & 2.9 \\
\hline Asp & 198 & 2.6 \\
\hline Asp & 199 & 2.7 \\
\hline Glu & 255 & 2.53 \\
\hline Glu & 260 & 2.58 \\
\hline Asp & 284 & 2.79 \\
\hline Glu & 386 & 2.52 \\
\hline Asp & 387 & 2.55 \\
\hline Glu & 389 & 2.58 \\
\hline Asp & 390 & 2.6 \\
\hline Glu & 396 & 2.72 \\
\hline Glu & 398 & 2.6 \\
\hline Glu & 399 & 2.73 \\
\hline Glu & 402 & 2.56 \\
\hline Glu & 403 & 2.7 \\
\hline Glu & 406 & 2.55 \\
\hline Leu & 417 & 2.85 \\
\hline
\end{tabular}

Extracted static PDB files from the simulation trajectory at timeframes of 10ns, 30ns and 50ns expounded that exon-9 in WT was unstable while WT-Ca showed a very stable assembly (Figure 9). The majority of residues that interacted with calcium were either glutamic acid or aspartic acid. The two alpha-helices in exon-9 of WT-Ca maintained a stable conformation of ' $\wedge$-shape' throughout the simulation. The two helices in exon- 9 are separated by an angle of $70.57^{\circ}$ while in WT-Ca by $54.44^{\circ}$. Moreover, in the absence of calcium, the two helices were separated by a distance of $38.73 \AA$ while in the presence of calcium they were separated by $29.9 \AA$. The presence of calcium 
ions provoked the C-terminal region to be displaced towards the opposing sides as indicated by the angular displacement of the C-terminal region in WT and WT-Ca (Figure S10). This stability could only be attained due to the higher magnitude of interaction with calcium ions which holds the structure steadily.

\section{Exon-9 Mutation leads to Imbalance of Calcium Flux}

We then sought to explore the effect of exon-9 mutations on protein structure in vitro in order to understand if there are any significant changes in their calcium regulation and fluxes. The transcriptional factor family Nuclear factor of activated T-cells (NFAT) is known for its involvement in calcium regulation $[48,49]$ and thus we first tested their mRNA levels. To our surprise, three members of the NFAT family members, namely NFAT 1, 3 and 5 were increased significantly in exon-9 mutated clones suggesting an imbalance of cellular calcium levels (Figure 10F). In order to validate the above mentioned hypothesis we explored the difference between WT and exon-9 KO cells using fluorescent microscopy. WT and exon-9 mutated cells were incubated for 30 mins with the Fluo4-AM probe and calcium signals were recorded using the $488 \mathrm{~nm}$ fluorescence channel. Both CALR exon-9 KO clones showed a sharp decrease of a spontaneous Fluo4-AM signal around the plasma membrane / extracellular region in comparison of WT. CALR has been found to localize in the extracellular region [50] and thus in the WT a higher calcium signal is observed as exon-9 can buffer and hold calcium ions while in the mutated exon-9 no such signal is detectable. To our surprise, there was a huge difference between the fluorescence intensities of calcium ions in the WT and mutated counterparts (Figure 10G). In sum, our in-vitro results showed that exon-9 plays a very crucial role in calcium binding and any mutation can drastically effect the cellular calcium levels. 


\section{$\underline{\text { Discussion }}$}

Essential thrombosis (ET) belongs to a sub-category of myeloproliferative neoplasms (MPNs) which enhances the formation of blood clotting through an unknown mechanism. Studies have found that mutations in two genes are responsible for such disorder where Janus kinase 2 (JAK2) and CALR account for $50-60 \%$ and $40-50 \%$, respectively. Much is known about the JAK2 mechanism while the role of CALR remains obscure although it was discovered as a susceptible gene a long time ago [51]. CALR is a highly conserved protein bestowed mostly with negative charged amino acids such as aspartic acid (Asp) (13.2\%) and galutamic acid (Glu) (12.9\%) while exon-9 accounts for $21.2 \%$ and $37.90 \%$ (Figure S5D) of the two amino acids, respectively, a twofold increase in Asp and three-fold in Glu. MPN patients encompass heterogeneity specifically in exon-9 leading to loss-of-function mutation and thereby generating a novel C-terminal sequence. The wild type CALR protein localizes in the ER to ensure proper folding of glycoproteins as well as to maintain calcium homeostasis [52] . Unavailability of a three-dimensional structure provoked us to model the full-length structure in order to visualize the overall structural dynamics in the absence and presence of calcium ions. As discussed by Abreusan et al. [53], an alpha helix provides more stability and robustness to proteins endorsing the fact that three out of four alpha- helices of CALR are encoded by in exon-9 albeit the mutation frequency is highest in exon-9. Moreover, the protein derived from exon-9 exhibits a helix-loop-helix structure and almost all of the INDEL mutations in humans start from Gln365 [5] which lies in the loop region while variations among different species start from E383 which lies in the helix region (Figure S1). The INDELs lead to the distortion of the C-terminal domain into the P-domain making a closed conformation due to the loss of structural integrity in both mutated isoforms (Figure 2B-C \& 4B-C). These mutated structures are more stable due to less fluctuations in their $\mathrm{C}$ terminal region, also evidenced by their kinetic energy $(\mathrm{KE})$ and they could readily interact with other proteins due to higher potential energy (PE). However, in the presence of calcium ions the WT protein attains conformational stability and could 
interact more readily with other proteins indicating the need of calcium ions for proper functioning as indicated by higher KE and PE (Figures 2 D-E, 3, 4D-E and Table S4). This is in line with the previous studies performed by Akari et al. [54] as the CALR mutants could interact more firmly with the thrombopoietin (TPO) receptor activating JAK2 and its downstream molecules such as mitogen-activated protein kinase 3 (MAPK3 or ERK1) and Signal transducer and activator of transcription 5 (STAT5). The binding of mutant CALR to the TPO receptor could presumably activate platelet production; which is also a common phenotype in ET patients. A similar study was performed by Pronier et al. [55] as they traced the interacting partners of mutated isoforms identifying friend leukemia integration 1 (FLI1) - a transcriptional regulator, as the key candidate that activates the TPO receptor and increases megakaryocytes formation which could be responsible for higher platelet production. Interestingly, Elf et al. had shown that mutant CALR binds to the TPO receptor which triggers MPN progression [56]. Thus, the modified C-terminal region in CALR proteins derived from mutant gnes might play a vital role in such interaction. Moreover, a direct study by Kollmann et al. [57] states that mutant CALR facilitates megakaryocyte production by MAPK signaling requiring calcium ions $[58,59]$. Apart from this, a recent study from Shide et al. [60] reported that homozygous mutations of $C A L R$ in mice result in mild thrombocytosis suggesting that a mutant $\mathrm{C}$-terminus can undergo numerous modifications depending on the position of the mutation and involved amino acids. A possible explanation could be that the CALR sequence in the C-terminus varies between human and mouse, giving rise to a different mutated C-terminal region with different binding propensities. Comparing human and mouse amino acid sequences, a few important amino acid substitutions like E60L in the P-domain, E402del in the C-terminal domain and V409S could play a vital role as in humans these residues are critical for providing stability in presence of calcium (Figures 1, 8, S7B and Table3). These variations in amino acids could differ in calcium binding strength which could give rise to a non-interacting and nonfunctional C-domain. 
The presence of calcium in the system provided clues that only the WT can bind to calcium ions due to the electrostatic interactions while INDELs could not as they lacked the negative C-terminus. The negatively charged environment in exon- 9 is able to compensate for the higher calcium load and maintained its conformational stability (Figures 5,8 ). $27.5 \%$ of total calcium ions remained bound to the C-terminal region while only $6.7 \%$ (Figure 8 ) accounted for the WT indicating that the C-terminal region is preferred by $\mathrm{Ca}^{2+}$ ions and not $\mathrm{Na}^{+}$ions. Our study also shed light on demonstrating that a monovalent ion like $\mathrm{Na}^{+}$is not enough to counter-balance the negative potential of the C-terminal region. Further studies are required in order to understand whether other divalent or trivalent ions could also provide such stability and interaction. An important observation in the current study is that conformational changes were not limited to exon-9, but the stability in exon-9 modulated the distally located P-domain in response to calcium binding. The P-N domain attained its structural stability due to the hydrogen bonds between His241-Gly140 and Ile242-Gly140 while the interaction of the C-N domain was contributed by the acidic residues Glu386, Glu389, Glu60 and Asp59 (Figure.8).

One of the key structural changes observed between WT and mutated proteins is the displacement of the second helix in the C-terminal domain (Figure S10) where helix 1 and helix 2 of the WT makes an angular displacement of $70.57^{\circ}$ and are separated by a distance of $38.73 \AA$ while in WTCa the two helices are much closer to each other with a distance of $29.99 \AA$ and an angular displacement of $54.44^{\circ}$ (Figure S10) . In the absence of calcium ions, WT and mutated counterparts reflected an opposite pattern of folding. WT remained in open configuration while mutated ones in closed indicating that the proteins derived from mutants have a very different architecture of amino acids which could trigger a cascade of reactions involved in calcium signaling as well as platelet formation causing diseases like ET. Although the mutations increased the structural stability, they lose their functionality as loss of the KDEL motif from the exon- 9 would remove the ER signature and thus they could no longer function as an ER chaperone and would localize to other 
cellular compartments. This loss of the ER retention signal would increase the calcium potential thereby disrupting the natural $\mathrm{pH}$ of 7.2 in the ER [61] and increasing an acidic ER stress. Moreover, recently it was reported that CALR could buffer $\sim 50 \%$ of ER calcium suggesting a very high level of calcium influx could be generated due to improper buffering [9].

CALR acts as a double edged sword. In one way, WT CALR plays a crucial role in buffering cellular calcium load due to the presence of a highly acidic C-terminal domain, and in return calcium ions provide structural stability to CALR by balancing the electrostatic charges. In contrast, a mutated CALR structure has a higher binding affinity for the TPO receptor that activates platelet-related transcription factors, as calcium plays a vital role in the production of platelets. In a wider sense, the current study touches upon the possible role of calcium ions in the formation of ET, although the sequences in the middle of exon-9 are evolutionary not conserved (Figure S1). One possible explanation could be that exon-9 was merged with CALR by natural selection to maintain calcium load and homeostasis only when calcium ions were available for uptake and utilization. The higher negative charge in exon-9 compared to other exons would help to effectively utilize calcium ions by neutralizing and balancing calcium charges. Exon-9 structural dynamics shows that gaining and loosing amino acids in the derived proteins by mutations significantly affects the structure which in turn would affect function (Figure S11). Overall, the findings from this study suggest a loss-offunction mutation in exon-9 of $C A L R$ which drives the onset of essential thrombocythemia through the imbalanced calcium load. Our result provides new structural insights into the working mechanism of intrinsically disordered $C A L R$ exon-9. We identified key residues involved in domain stability. Prospects lie in conducting experiments for $\mathrm{pH}$-dependent simulations in order to examine the correct physiological $\mathrm{pH}$ for CALR to be functional in the presence of calcium. It is also worth investigating possible key residues involved in the interaction between mutant CALR and the TPO receptor which will help in the screening of inhibitors to act as an antagonist for JAK2 activation. Calcium also acts in cell-to-cell communication and any change in the calcium level would trigger 
a response [62] in controlling a spectrum of cellular processes through calcium signal transduction [63]. Calcium ions are also essential for protein function as they can change protein shape and electrostatic charge distribution [18]. Hence, the protein derived from exon-9 being highly electronegative in nature, can easily bind and stabilize the charge of positive calcium ions. Exon-9 mutations imbalance the calcium homeostasis as evidenced from results highlighted in Figure 10 (G). WT cells have much higher calcium levels as CALR binds and sequesters calcium ions, while in contrast, mutant CALR is not able to hold calcium ions leading to very low cellular calcium levels. This is in accordance with a previous study where the authors showed that non-endoplasmic reticulum based CALR can affect calcium signaling [9]. Decreased extracellular calcium level hampers cell communication and finally leads to slower cell growth as imbalance of calcium ions could induce the G0 phase of the cell cycle [64]. This is in line with our data on slower cell proliferation of exon-9 KO $293 \mathrm{~T}$ cells (data not shown). We also observed an increased mRNA level of NFAT1,3 and 5 in exon-9 KO 293T cells, probably because cells are in a hypo-calcium state and thus requiring a higher calcium flux to maintain cellular homeostasis (Figure 10F), which remains to be further investigated.

\section{Conclusion}

Although $40-50 \%$ of ET is caused due to the mutations in the CALR gene, the exact mechanism remains vague. To understand the mechanistic insights of CALR dynamics in disease progression and etiology, this study employed full length structures of three isoforms of CALR and investigated the dynamics and transitions based on standard MDS. Secondly, we also studied the effect of calcium dynamics on the isoforms of the proteins to understand the calcium dynamics in regulating CALR. RMSD, Rg and RMSF between the isoforms of CALR revealed that the WT protein attains a highly unstable conformation compared to the two mutational isoforms. Two domains, namely the proline-rich P-domain and C-terminal domain displayed disordered movements. However, in 
the presence of calcium ions, the WT protein surmises a more compact and stable conformation than its mutated counterparts as a result of lower residue fluctuations in $\mathrm{P}$ and $\mathrm{C}$-terminal residues. Electrostatic effects mediated by the highly negative C-terminal domain attract free calcium which significantly decreases the enhanced fluctuations present in the C-terminal region. The affinity of the C-terminal region towards calcium ions further results in providing enhanced stability to the $\mathrm{N}$ terminal region as well. Thus, the presence and absence of calcium ions display contrasting behavior in the WT and mutated CALR. The mutated isoforms lack the electrostatic ability due to the absence of negative amino acids resulting in ineffective binding to calcium ions thereby perturbing the cellular calcium flux. We uncovered that the P-domain and C-terminal domain undergoe structural changes in the presence of calcium ions, thereby maintaining structural stability which can then interact with other proteins. The negative potential of the exon-9-drived protein alone is crucial for influencing stability and calcium homeostasis. Finally, Fluo4-AM staining indicated a huge difference in calcium intensity between WT and exon-9 KO cells. Our results should facilitate an understanding of ET formation via CALR mediated calcium signaling and might assist in finding therapeutic interventions for treating ET.

\section{Materials and Methods}

\section{Sequence analysis}

The protein sequence of $C A L R$ with the accession number P27797 was retrieved from Uniprot database (UniProt, 2015) in Fasta format, and the coding DNA sequence (CDS) with ID of ENSP00000320866.4 was retrieved from the Ensembl database [24]. According to a previous annotation [5], a 52 base pair deletion (Del) and 5 base pair insertion (Ins) were manually manipulated in the coding region of exon-9 in-frame to mimic $C A L R$ mutations. The secondary structures of the wild type and mutated CALR were examined by the PDBsum web server [25]. CALR domains were characterized by using the Pfam [26], InterProScan [27] and Uniprot webservers, and the physiochemical property of $C A L R$ was examined with the Protparam tool [28]. 


\section{Structural characterization}

Currently only a limited part of the CALR structure could be resolved [29]. The I-TASSER package [30] was used to construct the full-length CALR structure through homology modelling based on the multiple threading alignment by LOMETS [31] and the iterative TASSER [32] assembly simulations. The CALR mutant structures were constructed similarly based on the INS and DEL sequences. The built models were evaluated using the PROCHECK server [33], VERIFY3D [34], and ERRAT web servers [35]. The best-performed structures were firstly energy minimized using the steepest descent method to remove bad contacts within atoms using the SwissPDB viewer [36] and then subjected to a molecular dynamics (MD) simulation.

\section{Molecular Dynamics Simulation}

To characterize $C A L R$ and identify the role of $\mathrm{Ca}^{2+}$, molecular dynamics (MD) simulations on the WT (Wild Type) form as well as two mutant-forms namely Ins (inserted mutant) and Del (deleted mutant) together with the protein-Ca complexes WT-Ca, Ins-Ca and Del-Ca were performed using the GROMACS 4.6.5 package [37] combined with Amber99sb-ildn force field [38]. Each model was immersed into the center of a triclinic box of extended simple point charge (SPC-E) water molecules [39] with a margin distance of 10.0 Å. Periodic boundary conditions were applied to all directions. To keep the system in charge neutrality, $\mathrm{Na}^{+}$and $\mathrm{Ca}^{2+}$ counter ions were added to the WT, Ins, and Del forms making two experimental groups - sodium bound group (WT$\mathrm{Na}$, Ins-Na, and Del-Na) systems and calcium bound groups (WT-Ca, Ins-Ca, and Del-Ca) systems, respectively. 30000 steps of steepest descent energy minimization (EM) and 20000 steps of conjugated gradient EM were conducted in order on the constructed models. Then each system was gradually heated from $0 \mathrm{~K}$ to $300 \mathrm{~K}$ under conserved number of moles, volume and temperature (NVT) ensemble over a period of 500 pico-seconds (ps), during which a weak constraint of 10 $\mathrm{kcal} \cdot \mathrm{mol}^{-1} \cdot \AA^{-2}$ was imposed onto the protein. Afterwards, each model was subjected to an equilibrium simulation for 1000 ps by removing all constraints. Finally, a production simulation for 
each model was conducted under conserved number of moles, pressure and temperature (NPT) ensemble. The reference temperatures were kept at 300K, the LINCS algorithm (Hess et al., 1997) for bond constraints was used. The MD trajectory was recorded every 2 ps. VMD [41] and Chimera [42] were applied for visualizing and analyzing the MD results.

\section{Cell Culture}

HEK-293T cells were cultured on cell culture dishes (Corning) and maintained in cell culture medium comprised of Dulbecco's modified Eagle's medium (Hyclone) supplemented with 10\% fetal bovine serum (FBS) (Sigma-Aldrich) and penicillin-streptomycin antibiotic solution (SigmaAldrich) under humidified conditions at $37^{\circ} \mathrm{C}, 5 \% \mathrm{CO}_{2}$.

\section{CRISPR plasmids preparation}

The CRISPR-Cas9 plasmid pSpCas9(BB)-2A-GFP (PX458) (Addgene plasmid \#48138) was used for generating the knockout mutants. Custom designed guide RNAs were synthesized (Generay, China), annealed and ligated into BbsI digested sites. The ligated products were transformed into chemically competent DH5 $\alpha$ E. coli cells (Transtaq, China). The ligated cells were then plated in Ampicillin selection plates $(100 \mu \mathrm{g} / \mathrm{mL}$ ampicillin $)$ and incubated at $37^{\circ} \mathrm{C}$ for $12-14$ hours. Single and isolated colonies were picked and expanded in LB medium containing ampicillin. Plasmid DNA was isolated using the Taraka Mini Plasmid Extraction Kit and insertion of guide RNAs was verified by Sanger sequencing using the universal forward primer for the human U6 promoter (5, GACTATCATATGCTTACCGT 3') (Biosune, China).

\section{Generation of a CALR mutant in HEK-293T cells}

To generate a CALR exon-9 mutant cell line, HEK-293T cells were first cultured in 24 well cell culture plates (NEST, China). The protocol by Ran at al. was used to generate the exon 9 KO cells [43]. After the cells attained 70\% confluency, 500 ng CRISPR-Cas9 (PX458) plasmid having the 
right sgRNA insert (Table S1) was transfected along with transfection reagent Lipofectamine 2000 (Sigma) at a 1:3 DNA to reagent ratio. Transfection medium was changed after 12 hours from transfection to the complete medium. Cells were harvested for fluorescence-activated cell sorting (FACS) (BD Biosciences) after 24 hours of incubation. As the plasmid was fused with GFP, GFP(+ve) cells were sorted in 96 well plate (Corning) and a total of 200 cells were sorted for each guide RNA. The medium was changed every five days and single clones were expanded for 21 days. The clones were picked according to confluency for further expansion, DNA was extracted using the Takara Universal Genomic Extraction Kit (TakaraBio) and was sent for sequence verification (Biosune, China).

\section{RNA Extraction and qPCR}

Total RNA was extracted using the TRIzol reagent (Invitrogen) according to the manufacturer's instructions. The RNA concentration was measured using a NanoDrop 8000 spectrophotometer (Thermo Scientific). 1 ug RNA was reverse transcribed using a PrimeScript RT-PCR kit (Takara Bio) by following the manufacturer's instructions. The cDNA was then employed for quantitative polymerase reaction (qPCR) using a SsoAdvanced ${ }^{\mathrm{TM}} \mathrm{SYBR}^{\circledR}$ Green Supermix (Bio-Rad, USA) with denaturation at $95{ }^{\circ} \mathrm{C}$ for $30 \mathrm{~s}$, followed by 40 cycles of denaturation at $95{ }^{\circ} \mathrm{C}$ for $15 \mathrm{~s}$, and annealing at $60{ }^{\circ} \mathrm{C}$ for $30 \mathrm{~s}$ in a CFX96 System (Bio-Rad). The primer sequences are listed in supplementary Table S1.

\section{Calcium imaging}

Cells were seeded on a glass cover slip chamber with $200 \mu$ l complete medium. The cells were grown until 70\% confluency and incubated with the calcium indicator Fluo-4 AM $(1 \mu \mathrm{M})$ (Invitrogen, US) for 30 minutes at room temperature. Images were captured using an inverted Zeiss LSM710 confocal microscope with a 40x oil immersion objective. A wavelength of $488 \mathrm{~nm}$ was used to excite 
the samples and fluorescence emission was collected at $>505 \mathrm{~nm}$ for single color calcium imaging.

\section{Statistical Analysis}

Statistical analysis between two groups was performed by Student's T-test using GraphPad Prism version 7 for Windows (GraphPad Software, La Jolla California, USA). A p-value of lower than 0.05 was considered significant.

\section{$\underline{\text { Abbreviations }}$}

\begin{tabular}{ll} 
ABPS & Adaptive Poisson-Boltzmann Solver \\
CALR & Calreticulin \\
Del & Deleted \\
ET & Essential Thrombocythemia \\
ER & Endoplasmic Reticulum \\
INDEL & Insertion and Deletion \\
Ins & Inserted \\
KE & Kinetic Energy \\
KO & Knock-out \\
MPN & Myeloid Proliferative Neoplasm \\
MDS & Molecular Dynamics Simulation \\
PE & Potential Energy \\
RMSD & Root Mean Square Displacement \\
RMSF & Root Mean Square Fluctuations \\
Rg & Radius of Gyration \\
WT & Wild type \\
\hline
\end{tabular}




\section{$\underline{\text { Data availability }}$}

Source data are provided for all experiments. Other data that support the findings of this study are available from the corresponding author, upon reasonable request.

\section{Competing interests}

The authors have no conflicts of interest to declare that are relevant to the content of this article.

\section{Acknowledgments}

The authors would like to thank Institute of Aging Research, Hangzhou Normal University for providing research facilities. We thank Dr. Gabriele Saretzki of Newcastle University for comments on the manuscript. Amit Jaiswal would also like to thank Ram Manohar Jaiswal and Pratima Jaiswal for their constant support throughout this research.

\section{$\underline{\text { Author Contributions }}$}

Conceptualization: Amit Jaiswal

Formal analysis: Amit Jaiswal, Zhiguo Wang

Figure Preparation: Amit Jaiswal

Funding acquisition: Zhenyu Ju, Zhiguo Wang

Investigation: Amit Jaiswal, Zhiguo Wang, Xudong Zhu, Zhenyu Ju

Methodology: Amit Jaiswal, Zhiguo Wang

Project administration: Amit Jaiswal, Zhiguo Wang, Zhenyu Ju

Writing original draft: Amit Jaiswal

Writing review \& editing: Zhiguo Wang, Xudong Zhu, Zhenyu Ju 


\section{Bibliography}

1. Beer PA, Green AR (2009) Pathogenesis and management of essential thrombocythemia. Hematology 2009:621-628. https://doi.org/10.1182/asheducation-2009.1.621

2. Vardiman JW, Thiele J, Arber DA, et al (2009) The 2008 revision of the World Health Organization (WHO) classification of myeloid neoplasms and acute leukemia: rationale and important changes. Blood 114:937-951. https://doi.org/10.1182/blood-2009-03-209262

3. Griesinger F, Hennig H, Hillmer F, et al (2005) A BCR-JAK2 fusion gene as the result of a $\mathrm{t}(9 ; 22)(\mathrm{p} 24 ; \mathrm{q} 11.2)$ translocation in a patient with a clinically typical chronic myeloid leukemia. Genes Chromosomes Cancer 44:329-333. https://doi.org/10.1002/gcc.20235

4. Sattler M, Griffin JD (2001) Mechanisms of transformation by the BCR/ABL oncogene. Int J Hematol 73:278-291

5. Nangalia J, Massie CE, Baxter EJ, et al (2013) Somatic CALR Mutations in Myeloproliferative Neoplasms with Nonmutated JAK2. N Engl J Med 369:2391-2405. https://doi.org/10.1056/NEJMoa1312542

6. Cao G, Kong J, Xing Z, et al (2018) Rapid detection of CALR type 1 and type 2 mutations using PNA-LNA clamping loop-mediated isothermal amplification on a CD-like microfluidic chip. Anal Chim Acta 1024:123-135

7. Gángó A, Mózes R, Boha Z, et al (2018) Quantitative assessment of JAK2 V617F and CALR mutations in Philadelphia negative myeloproliferative neoplasms. Leuk Res 65:42-48

8. Lu Y-C, Weng W-C, Lee H (2015) Functional Roles of Calreticulin in Cancer Biology. BioMed Res Int 2015:. https://doi.org/10.1155/2015/526524

9. Biwer LA, Good ME, Hong K, et al (2018) Non-Endoplasmic Reticulum-Based Calr (Calreticulin) Can Coordinate Heterocellular Calcium Signaling and Vascular Function. Arterioscler Thromb Vasc Biol 38:120-130

10. Guo L, Nakamura K, Lynch J, et al (2002) Cardiac-specific expression of calcineurin reverses embryonic lethality in calreticulin-deficient mouse. J Biol Chem 277:50776-50779

11. Ellgaard L, Riek R, Herrmann T, et al (2001) NMR structure of the calreticulin P-domain. Proc Natl Acad Sci 98:3133-3138

12. Nakamura K, Zuppini A, Arnaudeau S, et al (2001) Functional specialization of calreticulin domains. J Cell Biol 154:961-972

13. Afshar N, Black BE, Paschal BM (2005) Retrotranslocation of the chaperone calreticulin from the endoplasmic reticulum lumen to the cytosol. Mol Cell Biol 25:8844-8853

14. Bagur R, Hajnóczky G (2017) Intracellular Ca2+ sensing: role in calcium homeostasis and signaling. Mol Cell 66:780-788. https://doi.org/10.1016/j.molcel.2017.05.028 
15. Prins D, Michalak M (2011) Organellar Calcium Buffers. Cold Spring Harb Perspect Biol 3:a004069. https://doi.org/10.1101/cshperspect.a004069

16. Görlach A, Klappa P, Kietzmann DT (2006) The endoplasmic reticulum: folding, calcium homeostasis, signaling, and redox control. Antioxid Redox Signal 8:1391-1418

17. Ozcan U, Cao Q, Yilmaz E, et al (2004) Endoplasmic reticulum stress links obesity, insulin action, and type 2 diabetes. Science 306:457-461. https://doi.org/10.1126/science.1103160

18. Clapham DE (2007) Calcium Signaling. Cell 131:1047-1058. https://doi.org/10.1016/j.cell.2007.11.028

19. Marenholz I, Heizmann CW, Fritz G (2004) S100 proteins in mouse and man: from evolution to function and pathology (including an update of the nomenclature). Biochem Biophys Res Commun 322:1111-1122

20. Landstrom AP, Dobrev D, Wehrens XHT (2017) Calcium Signaling and Cardiac Arrhythmias. Circ Res 120:1969-1993. https://doi.org/10.1161/CIRCRESAHA.117.310083

21. Gardner J-A, Peterson JD, Turner SA, et al (2016) Detection of CALR Mutation in Clonal and Nonclonal Hematologic Diseases Using Fragment Analysis and Next-Generation Sequencing. Am J Clin Pathol 146:448-455. https://doi.org/10.1093/ajcp/aqw129

22. Klampfl T, Gisslinger H, Harutyunyan AS, et al (2013) Somatic mutations of calreticulin in myeloproliferative neoplasms. N Engl J Med 369:2379-2390

23. Consortium U (2015) UniProt: a hub for protein information. Nucleic Acids Res 43:D204D212

24. Hubbard T, Barker D, Birney E, et al (2002) The Ensembl genome database project. Nucleic Acids Res 30:38-41

25. Laskowski RA, Jab\lońska J, Pravda L, et al (2018) PDBsum: Structural summaries of PDB entries. Protein Sci 27:129-134

26. Finn RD, Bateman A, Clements J, et al (2014) Pfam: the protein families database. Nucleic Acids Res 42:D222-D230

27. Jones P, Binns D, Chang H-Y, et al (2014) InterProScan 5: genome-scale protein function classification. Bioinformatics 30:1236-1240

28. Gasteiger E, Hoogland C, Gattiker A, et al (2005) Protein identification and analysis tools on the ExPASy server. In: The proteomics protocols handbook. Springer, pp 571-607

29. Berman HM, Westbrook J, Feng Z, et al (2000) The protein data bank. Nucleic Acids Res 28:235-242

30. Zhang Y (2008) I-TASSER server for protein 3D structure prediction. BMC Bioinformatics 9:40

31. Wu S, Zhang Y (2007) LOMETS: A local meta-threading-server for protein structure 
prediction. Nucleic Acids Res 35:3375-3382. https://doi.org/10.1093/nar/gkm251

32. Wu S, Skolnick J, Zhang Y (2007) Ab initio modeling of small proteins by iterative TASSER simulations. BMC Biol 5:17. https://doi.org/10.1186/1741-7007-5-17

33. Laskowski RA, Rullmann JAC, MacArthur MW, et al (1996) AQUA and PROCHECK-NMR: programs for checking the quality of protein structures solved by NMR. J Biomol NMR 8:477486

34. Eisenberg D, Lüthy R, Bowie JU (1997) [20] VERIFY3D: assessment of protein models with three-dimensional profiles. In: Methods in enzymology. Elsevier, pp 396-404

35. Colovos C, Yeates TO (1993) ERRAT: an empirical atom-based method for validating protein structures. Protein Sci 2:1511-1519

36. Kaplan W, Littlejohn TG (2001) Swiss-PDB viewer (deep view). Brief Bioinform 2:195-197

37. Abraham MJ, Murtola T, Schulz R, et al (2015) GROMACS: High performance molecular simulations through multi-level parallelism from laptops to supercomputers. SoftwareX 1:1925

38. Lindorff-Larsen K, Piana S, Palmo K, et al (2010) Improved side-chain torsion potentials for the Amber ff99SB protein force field. Proteins Struct Funct Bioinforma 78:1950-1958

39. Berendsen HJC, Grigera JR, Straatsma TP (1987) The missing term in effective pair potentials. J Phys Chem 91:6269-6271. https://doi.org/10.1021/j100308a038

40. Hess B, Bekker H, Berendsen HJC, Fraaije JGEM LINCS: A linear constraint solver for molecular simulations. J Comput Chem 18:10

41. Humphrey W, Dalke A, Schulten K (1996) VMD: visual molecular dynamics. J Mol Graph $14: 33-38$

42. Pettersen EF, Goddard TD, Huang CC, et al (2004) UCSF Chimera - a visualization system for exploratory research and analysis. J Comput Chem 25:1605-1612

43. Ran FA, Hsu PD, Wright J, et al (2013) Genome engineering using the CRISPR-Cas9 system. Nat Protoc 8:2281-2308. https://doi.org/10.1038/nprot.2013.143

44. Mészáros B, Erd $\backslash H o s$ G, Dosztányi Z (2018) IUPred2A: context-dependent prediction of protein disorder as a function of redox state and protein binding. Nucleic Acids Res 46:W329W337

45. Ikai A (1980) Thermostability and aliphatic index of globular proteins. J Biochem (Tokyo) 88:1895-1898

46. Brosnan JT, Brosnan ME (2006) The sulfur-containing amino acids: an overview. J Nutr 136:1636S-1640S

47. Laskowski RA, Swindells MB (2011) LigPlot+: multiple ligand-protein interaction diagrams for drug discovery. ACS Publications 
48. Ho SN, Thomas DJ, Timmerman LA, et al (1995) NFATc3, a lymphoid-specific NFATc family member that is calcium-regulated and exhibits distinct DNA binding specificity. J Biol Chem 270:19898-19907

49. Hogan PG, Chen L, Nardone J, Rao A (2003) Transcriptional regulation by calcium, calcineurin, and NFAT. Genes Dev 17:2205-2232

50. Varricchio L, Falchi M, Dall'Ora M, et al (2017) Calreticulin: Challenges Posed by the Intrinsically Disordered Nature of Calreticulin to the Study of Its Function. Front Cell Dev Biol 5:. https://doi.org/10.3389/fcell.2017.00096

51. Ostwald TJ, MacLennan DH (1974) Isolation of a high affinity calcium-binding protein from sarcoplasmic reticulum. J Biol Chem 249:974-979

52. Wang W-A, Liu W-X, Durnaoglu S, et al (2017) Loss of Calreticulin Uncovers a Critical Role for Calcium in Regulating Cellular Lipid Homeostasis. Sci Rep 7:5941. https://doi.org/10.1038/s41598-017-05734-X

53. Abrusán G, Marsh JA (2016) Alpha helices are more robust to mutations than beta strands. PLoS Comput Biol 12:e1005242

54. Araki M, Yang Y, Masubuchi N, et al (2016) Activation of the thrombopoietin receptor by mutant calreticulin in CALR-mutant myeloproliferative neoplasms. Blood 127:1307-1316. https://doi.org/10.1182/blood-2015-09-671172

55. Pronier E, Cifani P, Merlinsky TR, et al (2018) Targeting the CALR interactome in myeloproliferative neoplasms. JCI Insight 3:

56. Elf S, Abdelfattah NS, Chen E, et al (2016) Mutant calreticulin requires both its mutant Cterminus and the thrombopoietin receptor for oncogenic transformation. Cancer Discov. https://doi.org/10.1158/2159-8290.CD-15-1434

57. Kollmann K, Warsch W, Gonzalez-Arias C, et al (2017) A novel signalling screen demonstrates that CALR mutations activate essential MAPK signalling and facilitate megakaryocyte differentiation. Leukemia 31:934-944

58. Di Buduo CA, Moccia F, Battiston M, et al (2014) The importance of calcium in the regulation of megakaryocyte function. Haematologica 99:769-778

59. Kostyak JC, Naik MU, Naik UP (2012) Calcium-and integrin-binding protein 1 regulates megakaryocyte ploidy, adhesion, and migration. Blood J Am Soc Hematol 119:838-846

60. Shide K, Kameda T, Kamiunten A, et al (2019) Mice with Calr mutations homologous to human CALR mutations only exhibit mild thrombocytosis. Blood Cancer J 9:1-9

61. Wu MM, Llopis J, Adams S, et al (2000) Organelle pH studies using targeted avidin and fluorescein-biotin. Chem Biol 7:197-209

62. Bouschet T, Henley JM (2005) Calcium as an extracellular signalling molecule: perspectives on the Calcium Sensing Receptor in the brain. C R Biol 328:691-700. https://doi.org/10.1016/j.crvi.2004.10.006 
bioRxiv preprint doi: https://doi.org/10.1101/2021.08.05.455248; this version posted August 6, 2021. The copyright holder for this preprint (which was not certified by peer review) is the author/funder. All rights reserved. No reuse allowed without permission.

63. Fedrizzi L, Lim D, Carafoli E (2008) Calcium and signal transduction. Biochem Mol Biol Educ Bimon Publ Int Union Biochem Mol Biol 36:175-180. https://doi.org/10.1002/bmb.20187

64. Berridge MJ (1995) Calcium signalling and cell proliferation. BioEssays News Rev Mol Cell Dev Biol 17:491-500. https://doi.org/10.1002/bies.950170605 
bioRxiv preprint doi: https://doi.org/10.1101/2021 08.05.455248; this version posted August 6, 2021. The copyright holder for this preprint (which was not certified by peer review) is the author/funder. All rights reserved. No reuse allowed without permission.

\section{Figures}

Figure 1 Gene-loci, Transcript, Sequence and Structural information of Calreticulin

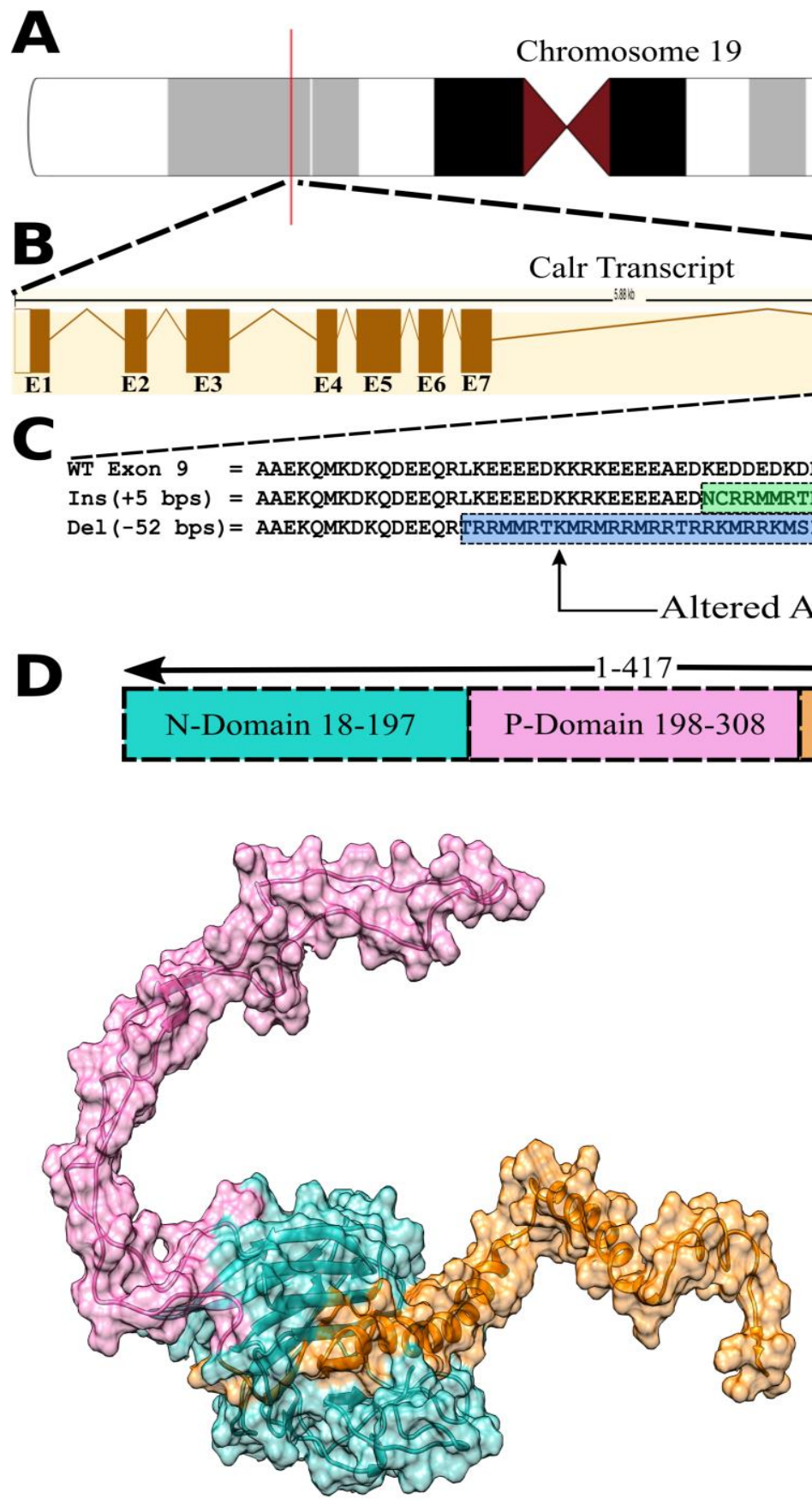
C-Domain 309-417
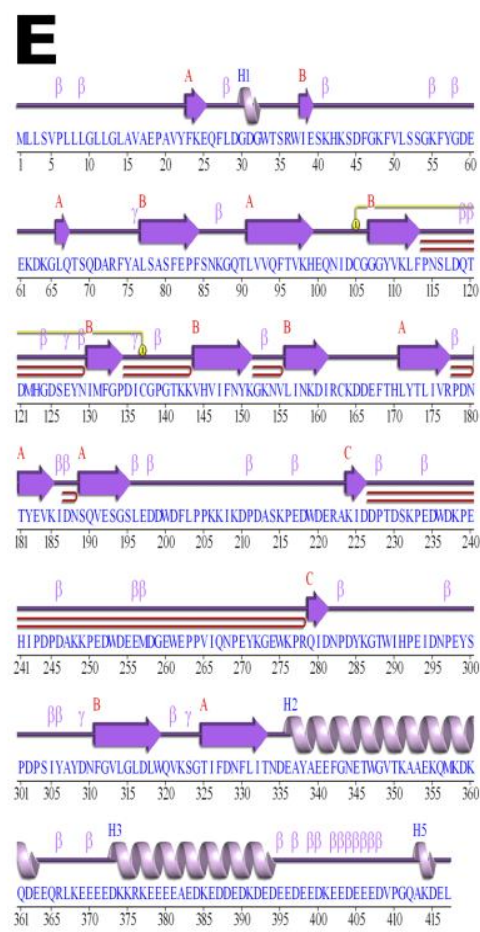
Figure 2:Comparison of WT, Ins and Del based on structure, RMSD and Rg in absence of calcium

A

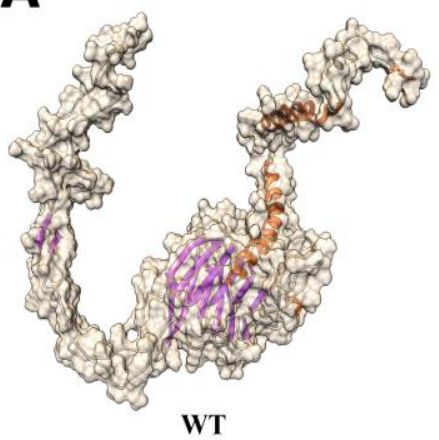

D

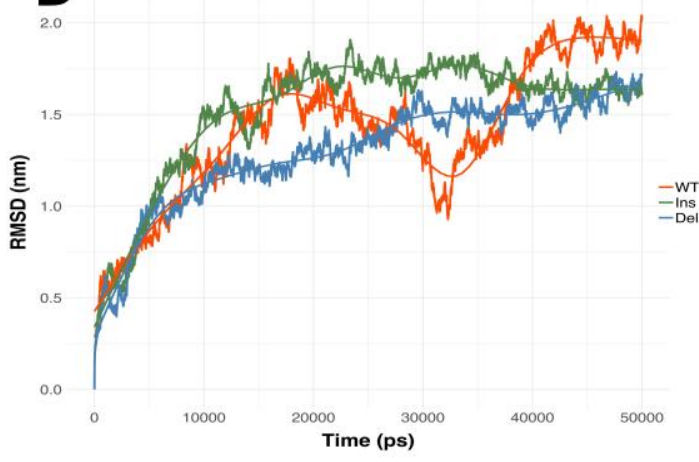

B

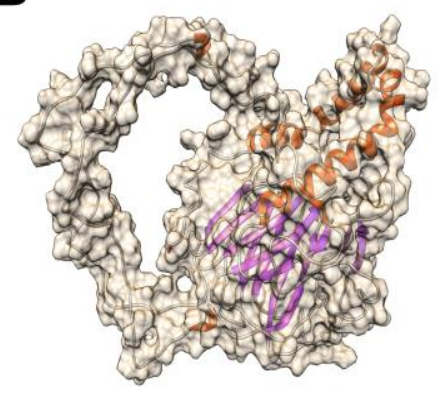

Ins
C

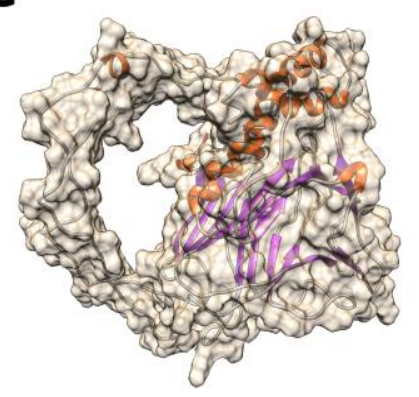

Del

E

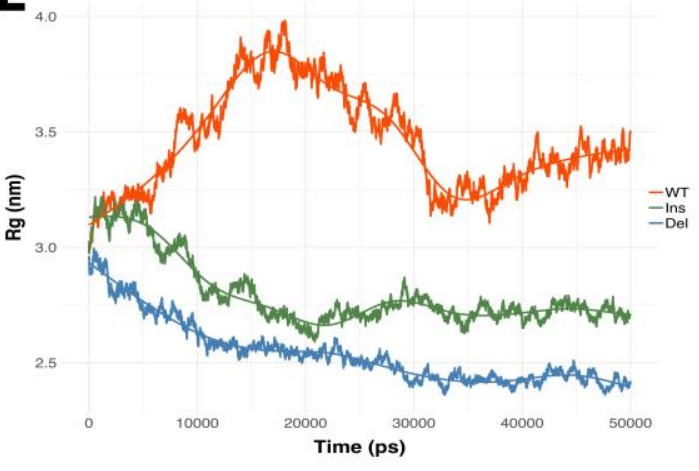


Figure 3: Comparison of RMSF in presence and absence of calcium ions where higher peak indicates higher residue fluctuations
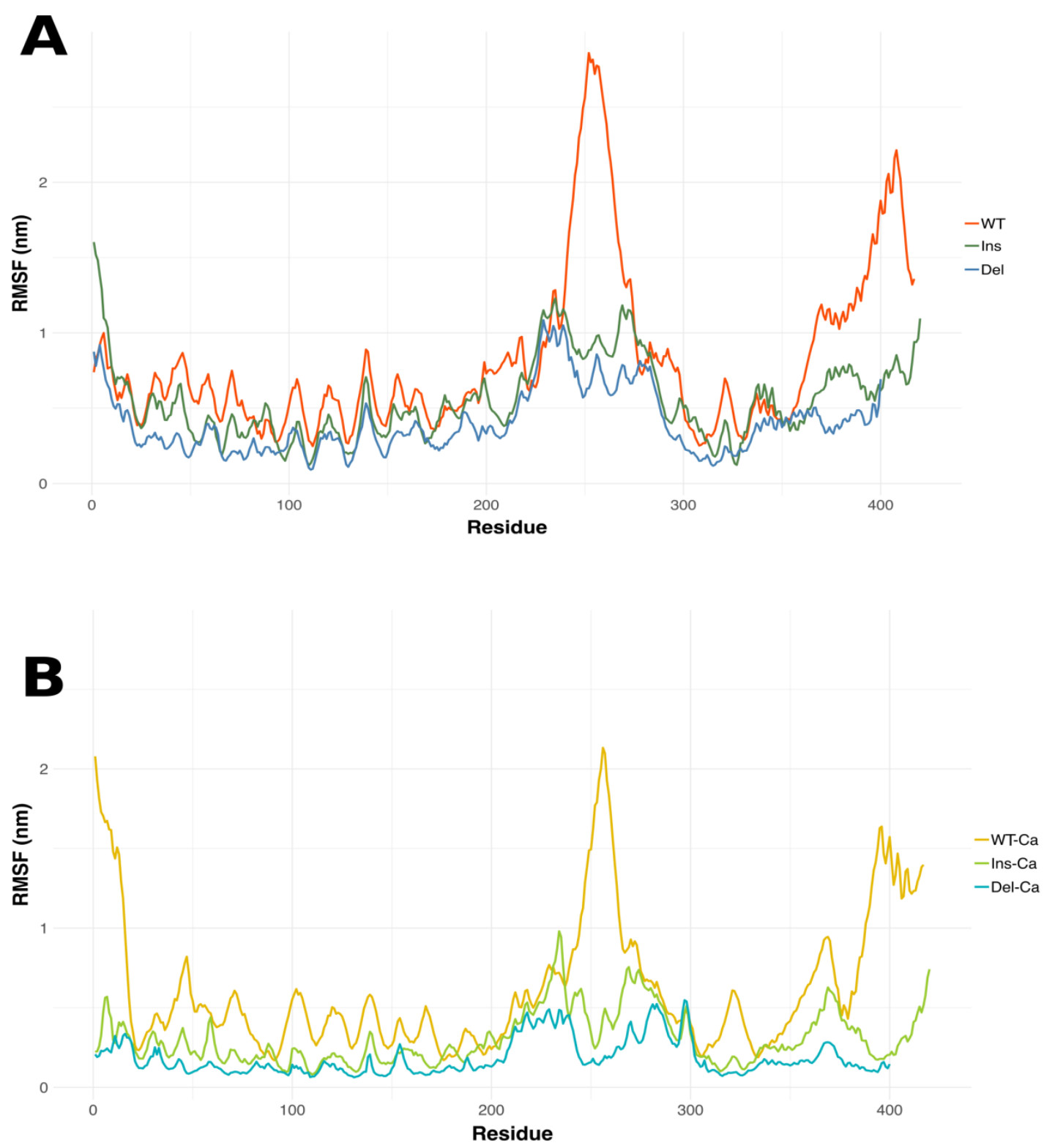
bioRxiv preprint doi: https://doi.org/10.1101/2021.08.05.455248; this version posted August 6, 2021. The copyright holder for this preprint (which was not certified by peer review) is the author/funder. All rights reserved. No reuse allowed without permission.

Figure 4 Comparison of WT-Ca, Ins-Ca and Del-Ca based on structure, RMSD and Rg in presence of calcium

A

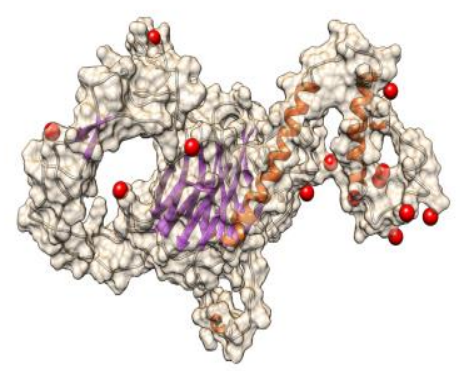

WT-Ca
B

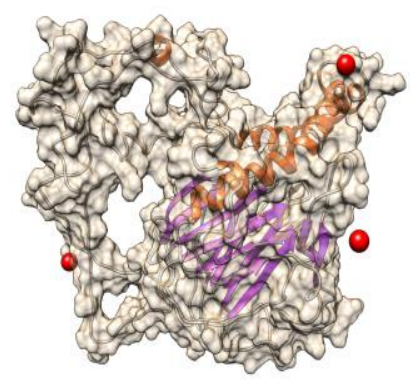

Ins-Ca
C

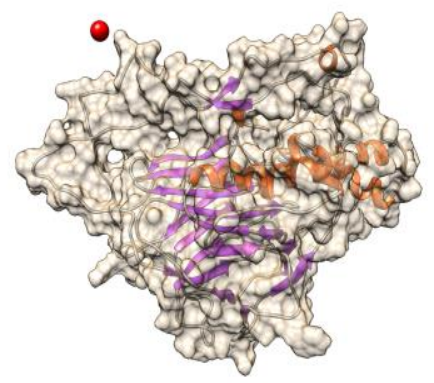

Del-Ca
D

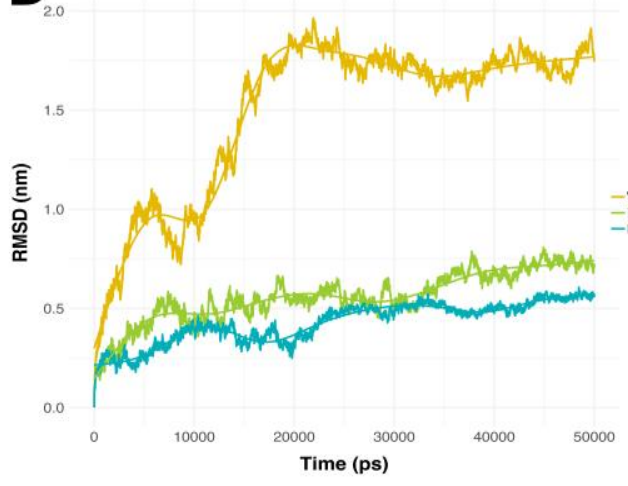

$\mathbf{E}$

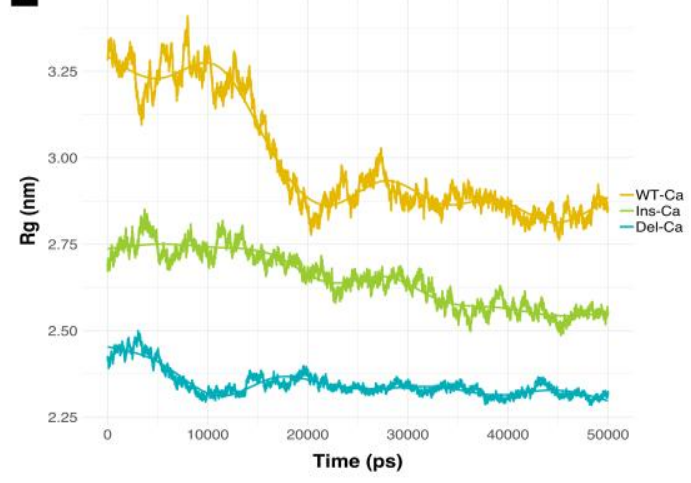


Figure 5 Structural alignment between proteins in presence and absence of calcium ions
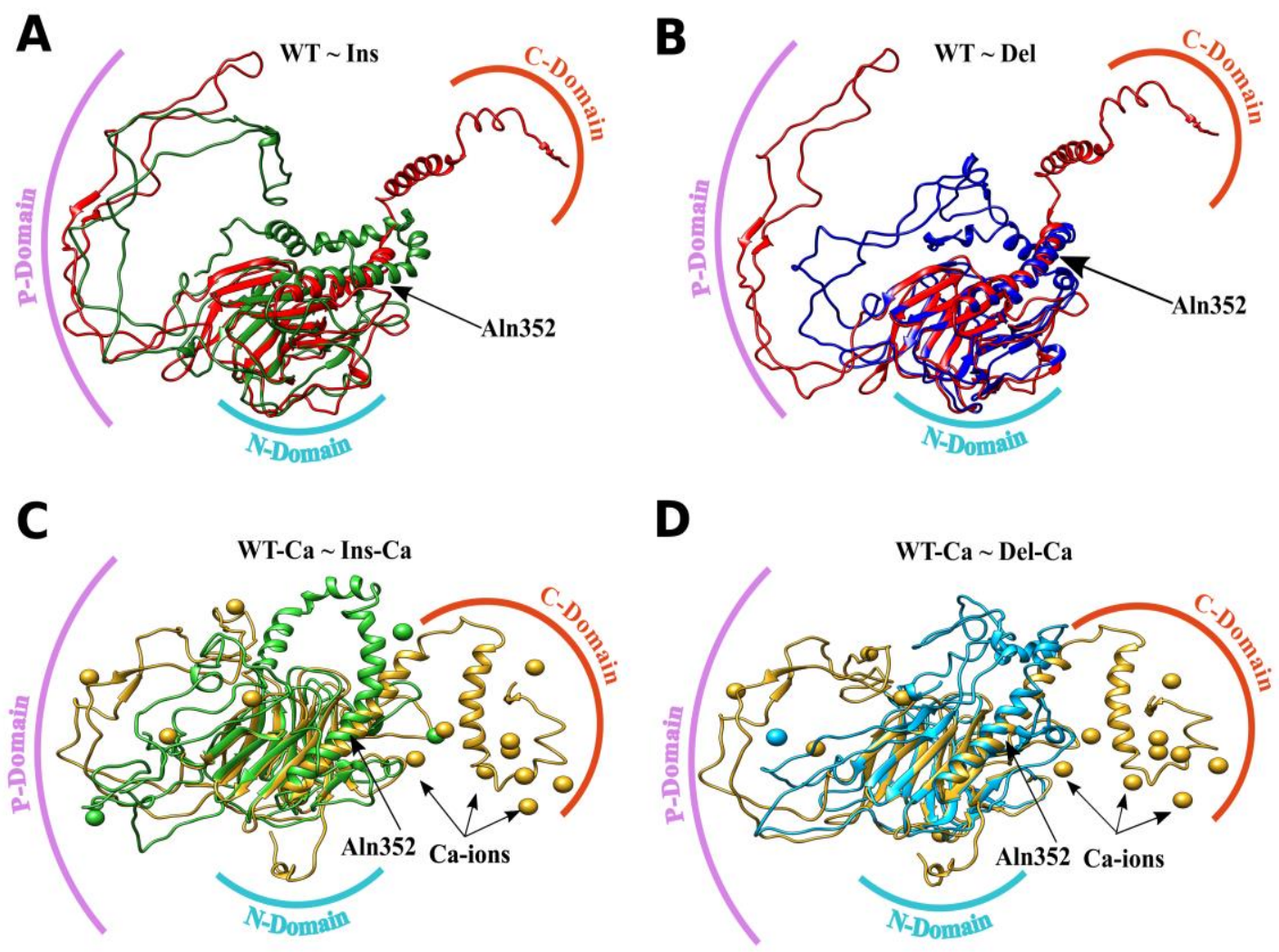
bioRxiv preprint doi: https://doi.org/10.1101/2021.08.05.455248; this version posted August 6, 2021. The copyright holder for this preprint (which was not certified by peer review) is the author/funder. All rights reserved. No reuse allowed without permission.

Figure 6 Structural changes of WT in presence and absence of calcium ions

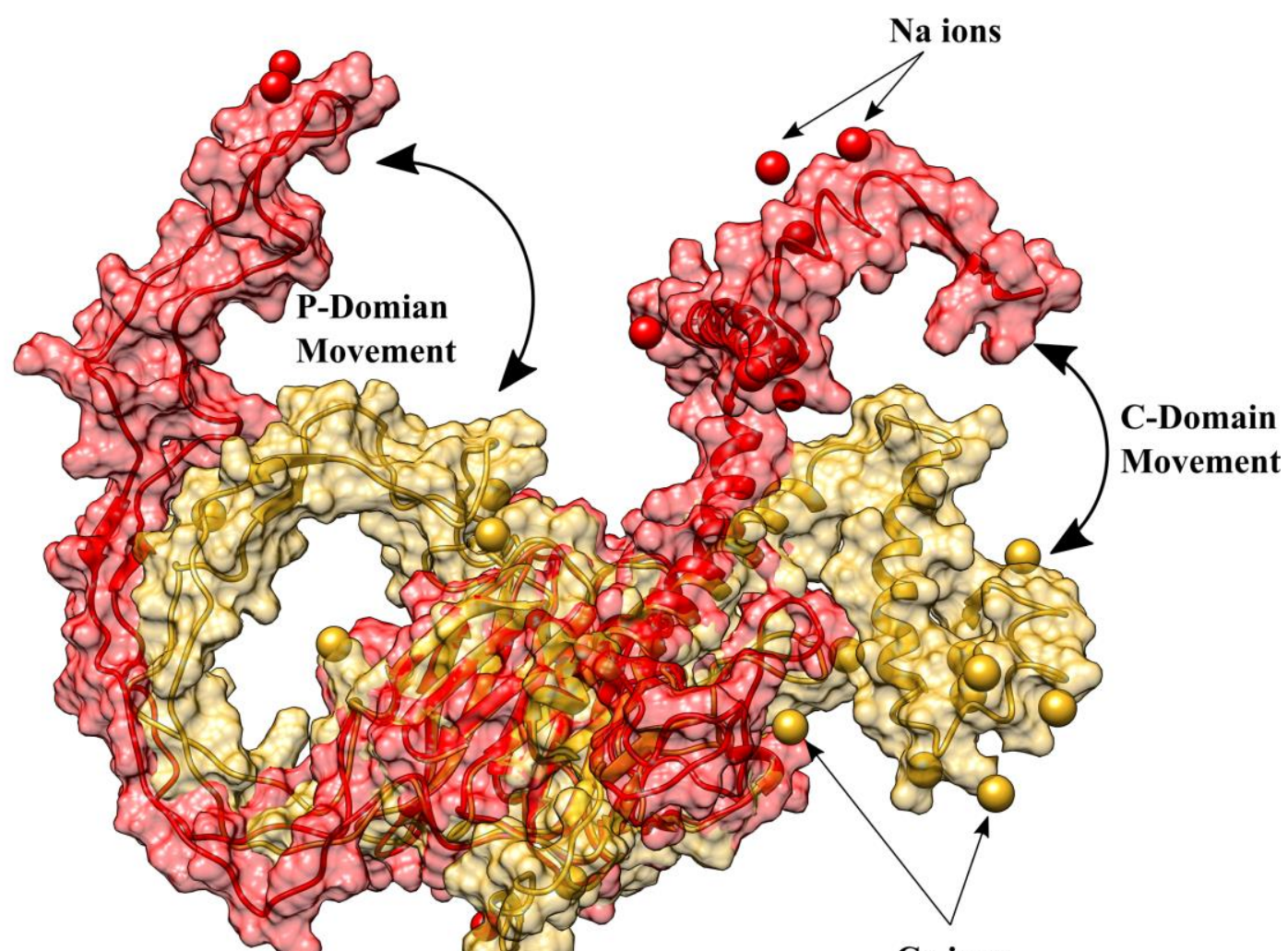

Ca ions 
bioRxiv preprint doi: https://doi.org/10.1101/2021 08.05.455248; this version posted August 6, 2021. The copyright holder for this preprint (which was not certified by peer review) is the author/funder. All rights reserved. No reuse allowed without permission.

Figure 7:Comparative analysis of calcium dynamics and electrostatics betweeen WT and WT-Ca
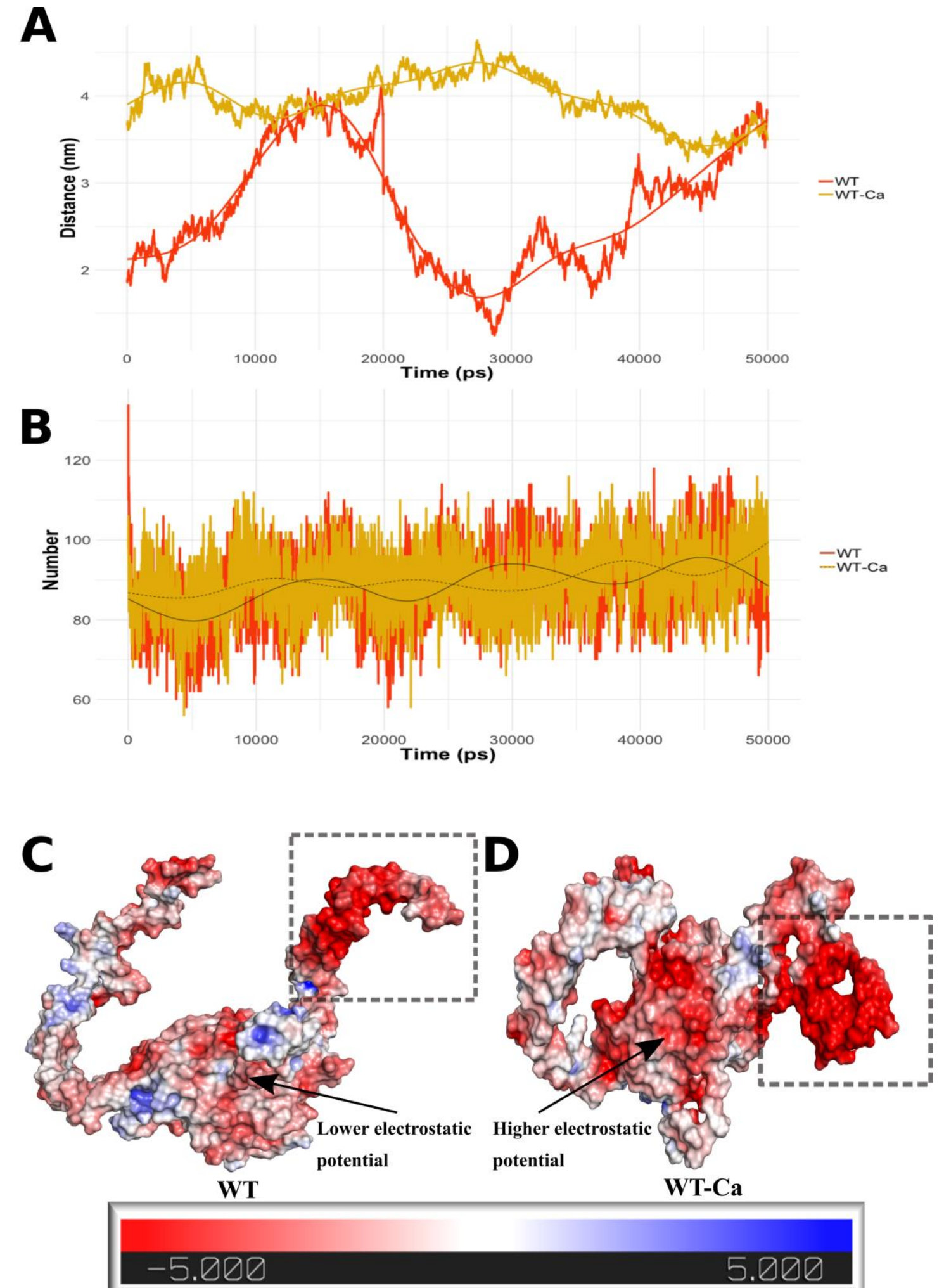
Figure 8 Interaction of various domains in presence of calcium ions

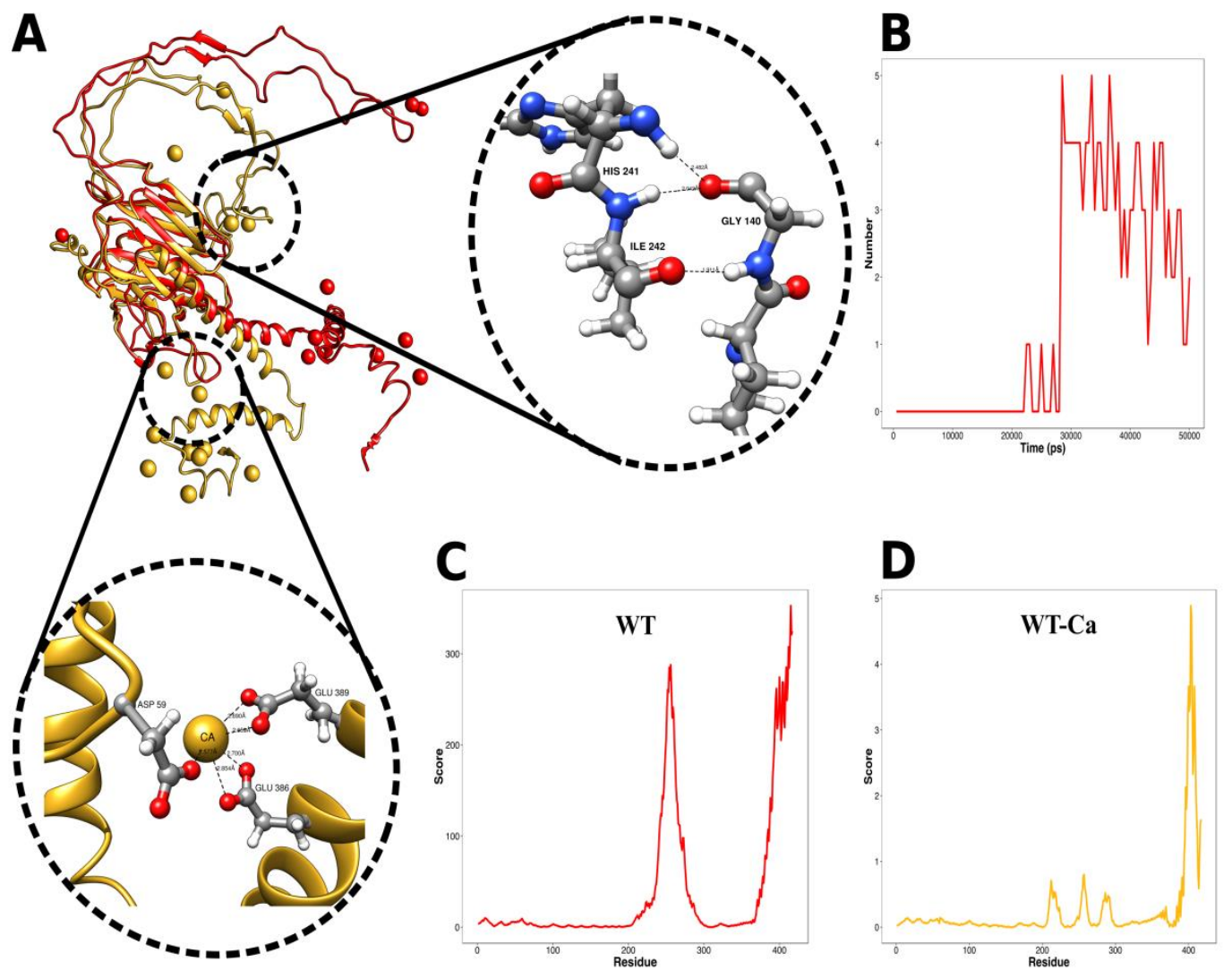


Figure 9 Time series Conformational changes in Exon-9 of WT and WT-Ca

A

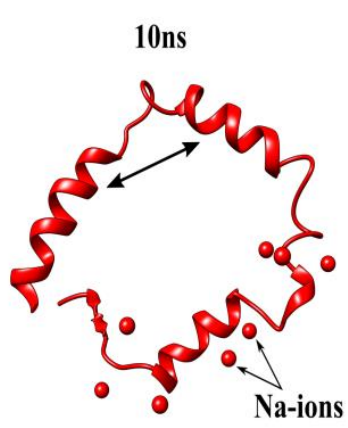

WT

D

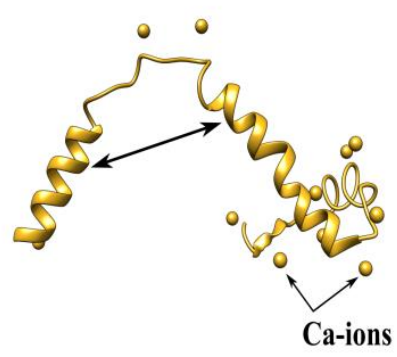

WT-Ca
B

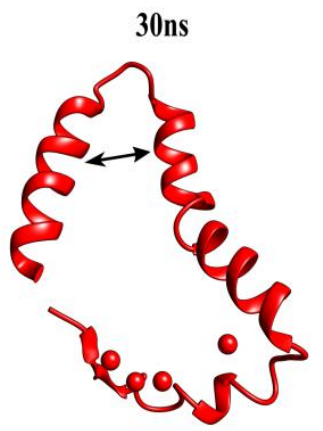

WT

E

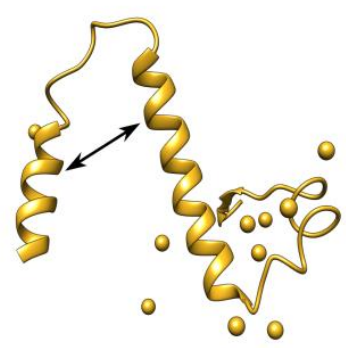

WT-Ca

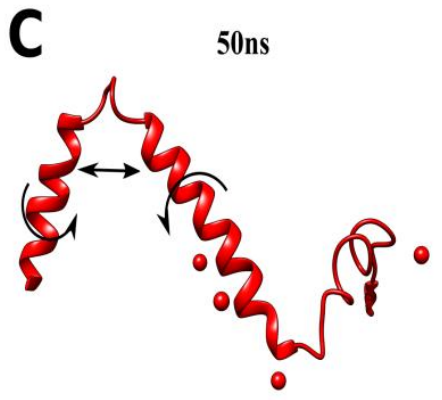

WT

F 50ns

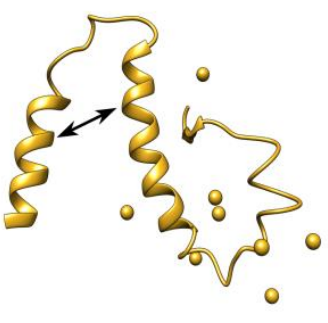

WT-Ca 


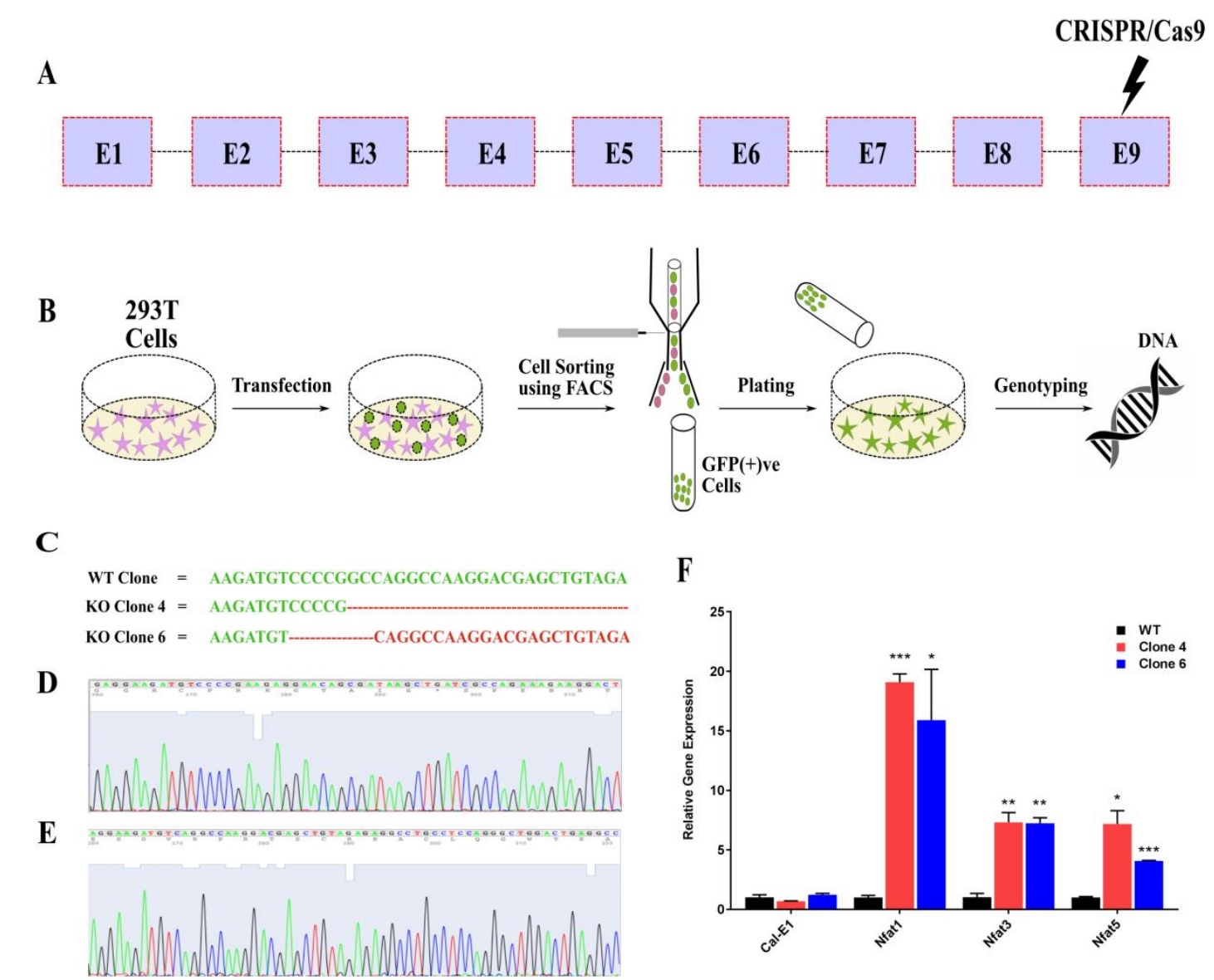

G

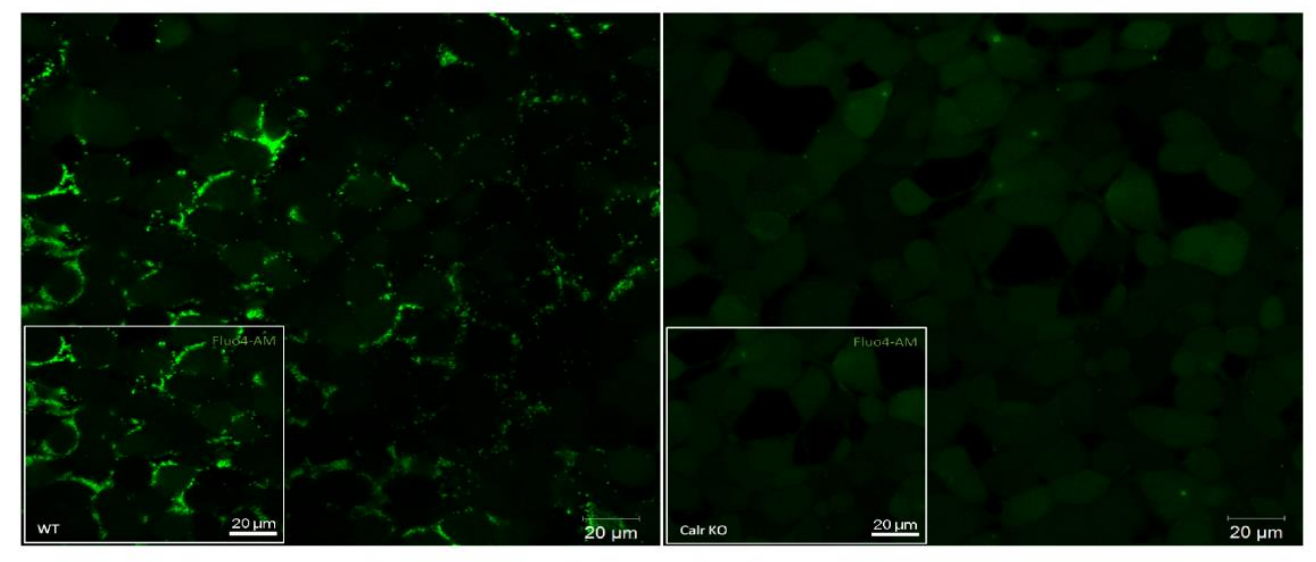




\section{Figure Legends}

Figure1: Gene locus transcript, sequence and structural information of calreticulin (CALR).

(A) Gene locus information of CALR. (B) Transcript information showing presence of nine exons.

(C) differences in the amino acid sequences between WT and mutants. Ins gains 5 bps in Exon-9 while Del loses 52 bps from exon-9. Altered amino acid sequence in Ins is highlighted in green color while in blue color in Del. (D) 3D structure of $C A L R$. The domains are coloured in different colours. $\mathrm{N}$ or globular domain is colored in sky-blue, P-domain in pink, C-domain in orange. (E) Secondary structure of $C A L R$. PDBsum webserver was used to prepare the figure.

Figure 2: Comparison of WT, Ins and Del based on structure, RMSD and Rg in absence of calcium.

(A), (B) and (C) represent 3D structures of WT, Ins and Del, respectively. The cartoon inside the surface view represents the secondary structure. Alpha-helices are colored in orange, beta-sheets in magenta. (D) Root mean square deviation (RMSD) of WT, Ins and Del in red, green and blue, respectively. (E) Radius of Gyration (Rg) of WT, Ins and Del (red, green and blue, respectively).

Figure 3: Comparison of RMSF in presence and absence of calcium ions where higher peak indicates higher residue fluctuations

(A) Root Mean Square Fluctuations (RMSF) in the absence of calcium. Red, green and blue color represent WT, Ins and Del, respectively. (B) RMSF in the presence of calcium. Golden, light-green and sky-blue represent WT-Ca, Ins-Ca and Del-Ca, respectively.

Figure 4: Comparison of WT-Ca, Ins-Ca and Del-Ca based on structure, RMSD and Rg in presence of calcium. 
(A), (B) and (C) represent 3-D structures of WT-Ca, Ins-Ca and Del-Ca. The cartoon representation inside the surface view is based on secondary structure. $\alpha$-Helices are colored in orange, $\beta$-sheets in magenta. (D) RMSD of WT-Ca, Ins-Ca and Del-Ca colored in golden, light-green and sky-blue. (E) Rg of WT-Ca, Ins-Ca and Del-Ca colored in golden, light-green and sky-blue.

\section{Figure 5: Structural alignment between proteins in presence and absence of calcium ions}

(A) and (B) structural alignment of WT with Ins and Del in absence of calcium ions. (C) and (D) the same as in (A) and (B) but in presence of calcium ions. WT (in red), Ins and Del are colored in green and blue. WT-Ca, Ins-Ca and Del-Ca are colored in golden, light-green and sky-blue. The starting residue for exon-9 is indicated as Aln352. Various protein domains are colored in various colors. The P-domain is indicated in pink, N-domain in blue and C-domain in orange color while calcium ions are represented as golden balls.

\section{Figure 6: Structural changes of WT in presence and absence of calcium ions}

3D structure of WT in absence of $\mathrm{Ca}^{++}$ions is shown in Red while 3D structure of WT in presence of $\mathrm{Ca}^{++}$ions (golden.) Both structures were superimposed to each other. The structural variation and movement of $\mathrm{P}$ and $\mathrm{C}$-domain are represented by arrows.

Figure 7: Comparative analysis of calcium dynamics and electrostatics between WT and WT-

\section{Ca}

(A) minimum distance of ions from exon-9. Red color: distance of $\mathrm{Na}^{+}$ions from WT. Golden: distance of calcium ions from WT-Ca. (B) number of intra-hydrogen bonds between WT and WTCa. Red: WT. Golden: WT-Ca. (C) electrostatic potential in WT. (D) electrostatic potential in WTCa. Red: higher electrostatic potential, blue: lower electrostatic potential. Areas shown with arrows and highlighted with dashed lines indicate differences in electrostatic charges. 


\section{Figure 8: Interaction of various domains in presence of calcium ions.}

(A) key residues responsible for the interaction of N-domain to P and C domain. WT (red) has no domain interaction while WT-Ca (golden) undergoes structural transformation through the domain interaction. N-P domain is stabilized due to the interaction between Gly140with His241 and Ile242. N-C domain is stabilized due to the interaction of Asp59, Glu60, Glu386 and Glu389 in presence of Calcium ions represented in golden color. The red balls represent oxygen atoms while the white balls represent hydrogen atoms, the blue balls represent nitrogen and the grey color represents carbon backbone. (B) formation of hydrogen bonds between N-P domain throughout simulation. (C) and (D) represent the mobility of WT in presence and absence of calcium ions.

\section{Figure 9: Time series Conformational changes in Exon-9 of WT and WT-Ca.}

(A-C) conformational changes and local transformations in exon-9 in the absence of calcium with time lapse of 10ns, 30ns and 50ns (D-F) conformational changes and local transformations in exon9 in the presence of calcium with time lapse of 10ns, 30ns and 50ns.

\section{Figure 10: In-vitro results of WT and Mutated $C A L R$}

(A) Gene map of CALR (B) generation of CALR exon-9 mutations using CRISPR-Cas9 (C) sequence analysis of mutated clones taken under investigation (D)-(E) Sanger sequencing results of the mutated clones compared to WT (F) qPCR results of CALR Exon-1, NFAT1, NFAT3 and NFAT5 in WT and two mutated clones: clonec 4 and clone 6 where WT is depicted in black color, clone 4 in red and clone 6 in blue respectively $(\mathrm{G})$ representative images of Fluo4-AM staining indicating the fluorescence intensity of calcium ions in WT and mutated counterparts. 